\title{
Gabor Expansions and Restricted Summability
}

\author{
Ferenc Weisz* \\ Department of Numerical Analysis, Eötvös L. University \\ H-1117 Budapest, Pázmány P. sétány 1/C., Hungary \\ e-mail: weisz@numanal.inf.elte.hu
}

\begin{abstract}
A general summability method, the so called $\theta$-summability is considered for Gabor series. It is proved that the maximal operator of the $\theta$-means defined in a cone is bounded from the local Hardy space $h_{p}$ to $L_{p}$ and from the amalgam space $W\left(h_{p}, \ell_{\infty}\right)$ to $W\left(L_{p}, \ell_{\infty}\right)$. This implies the almost everywhere convergence of the $\theta$-means for all $f \in W\left(L_{1}, \ell_{\infty}\right)$.
\end{abstract}

Key words and phrases: Wiener amalgam spaces, local Hardy spaces, atomic decomposition, $\theta$-summability, Gabor expansions, Gabor frames, time-frequency analysis.

2000 AMS Mathematics Subject Classification - Primary 42B08, 42C15, Secondary 42C40, 42A38, 46B15.

\section{Introduction}

Carleson's [4] famous theorem says hat the partial sums of the Fourier series of an $f \in L_{p}(\mathbb{T})(1<p<\infty)$ converge to $f$ almost everywhere. This is false for $p=1$. However, for Fejér, Riesz, Weierstrass, Abel, etc. summability methods, the corresponding means $\sigma_{n} f$ of the Fourier series of $f$ converge to $f$ almost everywhere if $f \in L_{1}(\mathbb{T})$ (see Zygmund [31], Butzer and Nessel [3], Stein and Weiss [23] or Trigub and Belinsky [25]).

A general method of summation, the so called $\theta$-summation method, which is generated by a single function $\theta$ and which includes all summations just mentioned, is studied intensively in the literature (see Butzer and Nessel [3], Trigub and Belinsky [25] and Weisz [27]). In Feichtinger and Weisz [7, 8] we have generalized these results and proved that if $\theta$ is in a Herz space then the maximal operator of the $\theta$-means $\sigma_{*}^{\theta}$ is bounded on $L_{p}(1<p \leq \infty)$ and of weak type $(1,1)$, i.e.,

$$
\sup _{\rho>0} \rho \lambda\left(\sigma_{*}^{\theta} f>\rho\right) \leq C\|f\|_{1} \quad\left(f \in L_{1}(\mathbb{T})\right) .
$$

${ }^{*}$ The Project is supported by the European Union and co-financed by the European Social Fund (grant agreement no. TAMOP 4.2.1/B-09/1/KMR-2010-0003). 
This implies the convergence of $\sigma_{n}^{\theta} f$ to $f$ a.e. as $n \rightarrow \infty$ and $f \in L_{1}(\mathbb{T})$.

We can generalize these results for multi-dimensional Fourier series basically in two different ways. The first one is if we define the $\theta$ function by $\theta(x):=$ $\theta_{0}(|x|),\left(x \in \mathbb{R}^{d}\right)$, where $|\cdot|$ denotes the Euclidean norm. Now we investigate the $\theta$-means $\sigma_{\mathbf{n}}^{\theta} f$ and the convergence over the diagonal $\mathbf{n}=(n, \ldots, n)$. In this case the analogous results can be found in Stein and Weiss [23] and Feichtinger and Weisz $[7,8]$. In the second, different way $\theta$ is given by the product of $d$ one-dimensional functions, $\theta(x):=\theta_{1}\left(x_{1}\right) \cdots \theta_{d}\left(x_{d}\right)$. Here we investigate the $\theta$ means and the convergence over a cone $\mathbb{N}_{\tau}^{d}:=\left\{n \in \mathbb{N}^{d}, 2^{-\tau} \leq n_{k} / n_{j} \leq 2^{\tau} ; k, j=\right.$ $1, \ldots, d\}$, where $\tau \geq 0$. For this type of summation the first and classical result is due to Marcinkiewicz and Zygmund [21]. They proved that the Fejér means (i.e., if each $\theta_{i}$ is the Fejér function) $\sigma_{n} f$ of a function $f \in L_{1}\left(\mathbb{T}^{d}\right)$ converge a.e. to $f$ as $n \rightarrow \infty$ provided that $n \in \mathbb{N}_{\tau}^{d}$. Moreover, the (restricted) maximal operator $\sigma_{*}$ defined in a cone is bounded from $H_{p}\left(\mathbb{T}^{d}\right)$ to $L_{p}\left(\mathbb{T}^{d}\right)$ whenever $d /(d+1)<p<\infty$ (see Weisz [27]).

In this paper we will consider the analogous results for summation of Gabor expansions

$$
\sum_{k, n \in \mathbb{Z}^{d}}\left\langle f, M_{\beta n} T_{\alpha k} g\right\rangle M_{\beta n} T_{\alpha k} \gamma
$$

of $f$, where $\alpha, \beta>0, g, \gamma \in W\left(L_{\infty}, \ell_{1}\right)\left(\mathbb{R}^{d}\right)$, and $M$ denotes the modulation operator and $T$ the translation operator. Here $W\left(L_{p}, \ell_{q}\right)\left(\mathbb{R}^{d}\right)$ denotes the Wiener amalgam spaces. We assume that the window functions $g$ and $\gamma$ generate dual Gabor frames for $L_{2}\left(\mathbb{R}^{d}\right)$. Gröchenig, Heil and Okoudjou [14, 15] proved the norm convergence of the partial sums of the Gabor series of $f \in W\left(L_{p}, \ell_{q}\right)\left(\mathbb{R}^{d}\right)$, $1<p<\infty, 1 \leq q<\infty$. The same result was also shown by Grafakos and Lennard [12] for $L_{p}\left(\mathbb{R}^{d}\right)(1<p<\infty)$ functions and for special window functions from the Schwartz class. If $\hat{\theta}$ is integrable and $1 \leq p<\infty$ then Feichtinger and Weisz $[9,6]$ verified the norm convergence of the $\theta$-summation method (see also Grafakos and Lennard [12] for the Fejér means). In the first case, i.e., if $\theta(x):=\theta_{0}(|x|)$, the almost everywhere convergence of the $\theta$-means of the Gabor series of $f \in W\left(L_{1}, \ell_{\infty}\right)\left(\mathbb{R}^{d}\right)$ and some inequalities concerning Hardy spaces can be found in $[30,29]$.

In the present paper we consider the second generalization of summation methods, when $\theta$ is the product of $d$ one-dimensional functions. Starting with the local Hardy spaces we introduce new Wiener amalgam spaces $W\left(h_{p}, \ell_{\infty}\right)\left(\mathbb{R}^{d}\right)$. Local Hardy spaces were introduced and investigated first by Goldberg [11]. These spaces has already been used also in Gabor analysis (see Gilbert and Lakey [10] and Weisz $[28,30]$ ). Under some conditions on $\theta$ we verify that the maximal operator $\sigma_{*}^{\theta}:=\sup _{n \in \mathbb{N}_{\tau}^{d}}\left|\sigma_{n}^{\theta}\right|$ of the $\theta$-means of Gabor series defined in a cone is bounded from the local Hardy spaces $h_{p}\left(\mathbb{R}^{d}\right)$ to $L_{p}\left(\mathbb{R}^{d}\right)\left(p_{0}<p<\infty\right)$ and from the Wiener amalgam Hardy space $W\left(h_{p}, \ell_{\infty}\right)\left(\mathbb{R}^{d}\right)$ to $W\left(L_{p}, \ell_{\infty}\right)\left(\mathbb{R}^{d}\right)\left(p_{0}<\right.$ $p<\infty)$. Because of the difference of the two types of summations, both proofs 
need essentially new ideas. By interpolation we obtain weak type inequalities which imply the analogue of the result due to Marcinkiewicz and Zygmund for Gabor series, i.e., $\sigma_{n}^{\theta} f$ converge a.e. to $f$ as $n \rightarrow \infty$ and $n \in \mathbb{N}_{\tau}^{d}$, provided that $f \in W\left(L_{1}, \ell_{\infty}\right)\left(\mathbb{R}^{d}\right)$. Finally, as special cases of the $\theta$-summation method we investigate some well known summability methods, such as the summation methods of Fejér, Riesz, Weierstrass, Abel, Picard, Bessel, Riemann, de La Vallée-Poussin and Rogosinski.

\section{Wiener amalgams and Hardy spaces}

Let us fix $d \geq 1, d \in \mathbb{N}$. For a set $\mathbb{Y} \neq \emptyset$ let $\mathbb{Y}^{d}$ be its Cartesian product $\mathbb{Y} \times \ldots \times \mathbb{Y}$ taken with itself d times. For $x=\left(x_{1}, \ldots, x_{d}\right) \in \mathbb{R}^{d}$ and $u=\left(u_{1}, \ldots, u_{d}\right) \in \mathbb{R}^{d}$ set $u \cdot x:=\sum_{k=1}^{d} u_{k} x_{k}$.

The norm (or quasi-norm) of the usual $L_{p}\left(\mathbb{R}^{d}\right)$ space is given by $\|f\|_{p}:=$ $\left(\int_{\mathbb{R}^{d}}|f|^{p} d \lambda\right)^{1 / p}(0<p \leq \infty)$, where $\lambda$ is the Lebesgue measure. The space of continuous functions with the supremum norm is denoted by $C\left(\mathbb{R}^{d}\right)$. We use the notation $|I|$ for the Lebesgue measure of the set $I$.

The weak $L_{p}$ space, $L_{p, \infty}\left(\mathbb{R}^{d}\right)(0<p<\infty)$ consists of all measurable functions $f$ for which

$$
\|f\|_{L_{p, \infty}}:=\sup _{\rho>0} \rho \lambda(|f|>\rho)^{1 / p}<\infty,
$$

while we set $L_{\infty, \infty}\left(\mathbb{R}^{d}\right)=L_{\infty}\left(\mathbb{R}^{d}\right)$. Note that $L_{p, \infty}\left(\mathbb{R}^{d}\right)$ is a quasi-normed space (see Bergh and Löfström [2]). It is easy to see that for each $0<p \leq \infty$,

$$
L_{p}\left(\mathbb{R}^{d}\right) \subset L_{p, \infty}\left(\mathbb{R}^{d}\right) \quad \text { and } \quad\|\cdot\|_{L_{p, \infty}} \leq\|\cdot\|_{p} .
$$

For a measurable function $\phi$ on $\mathbb{R}^{d}$ let

$$
\phi_{t}(x):=t^{-d} \phi(x / t) \quad\left(x \in \mathbb{R}^{d}, t>0\right) .
$$

Given a Schwartz function $\phi \in \mathcal{S}\left(\mathbb{R}^{d}\right)$ with $\int_{\mathbb{R}^{d}} \phi d \lambda \neq 0$ and $\operatorname{supp} \phi \subset[0,1 / 2]^{d}$, the local Hardy space $h_{p}\left(\mathbb{R}^{d}\right)(0<p \leq \infty)$ consists of all tempered distributions $f$ for which

$$
\|f\|_{h_{p}\left(\mathbb{R}^{d}\right)}:=\left\|\sup _{0<t<1}\left|f * \phi_{t}\right|\right\|_{L_{p}\left(\mathbb{R}^{d}\right)}<\infty .
$$

Taking the supremum over all $0<t<\infty$ we obtain the definition of the classical Hardy space $H_{p}\left(\mathbb{R}^{d}\right.$ ) (for more about classical Hardy spaces see Stein [22] or Weisz [27]). Other non-zero Schwartz functions $\phi$ define the same spaces and equivalent norms (resp. quasinorms for $p<1$ ). Usually the classical Hardy spaces are investigated. Local Hardy spaces were introduced in Goldberg [11] (see also Triebel [24]). They ensure a useful tool in Gabor analysis (see Gilbert and Lakey [10] and Weisz $[28,30])$. 
It is known that the Hardy spaces $h_{p}\left(\mathbb{R}^{d}\right), H_{p}\left(\mathbb{R}^{d}\right)$ are equivalent to the $L_{p}\left(\mathbb{R}^{d}\right)$ space when $1<p \leq \infty$ and $H_{1}\left(\mathbb{R}^{d}\right) \subset h_{1}\left(\mathbb{R}^{d}\right) \subset L_{1}\left(\mathbb{R}^{d}\right)$ (see e.g. Stein [22], Weisz [27] and Goldberg [11]).

The atomic decomposition is a useful characterization of Hardy spaces. A bounded function $a$ is a $h_{p}\left(\mathbb{R}^{d}\right)$-atom if there exists a cube $I \subset \mathbb{R}^{d}$ such that

(i) $\operatorname{supp} a \subset I$,

(ii) $\|a\|_{\infty} \leq|I|^{-1 / p}$,

(iii) if $|I|<1$ then $\int_{I} a(x) x^{k} d \lambda(x)=0$ for all multi-indices $k=\left(k_{1}, \ldots, k_{d}\right)$ with $|k| \leq L$, where $L \geq[d(1 / p-1)]$. Note that $[r]$ denotes the integer part of $r \in \mathbb{R}$.

We will say that $a$ is a type 1 atom if $|I|<1$ and a type 2 atom if $|I| \geq 1$. If we require the moment condition in (iii) for all intervals $I$ then we obtain the definition of $H_{p}\left(\mathbb{R}^{d}\right)$-atom (see Lu [20], Stein [22] and Goldberg [11]).

Theorem 1 A tempered distribution $f$ is in $h_{p}\left(\mathbb{R}^{d}\right)(0<p \leq 1)$ if and only if there exist a sequence $\left(a_{k}, k \in \mathbb{N}\right)$ of $h_{p}\left(\mathbb{R}^{d}\right)$-atoms and a sequence $\left(\mu_{k}, k \in \mathbb{N}\right)$ of real numbers such that

$$
\sum_{k=0}^{\infty}\left|\mu_{k}\right|^{p}<\infty \quad \text { and } \quad \sum_{k=0}^{\infty} \mu_{k} a_{k}=f \quad \text { in the sense of distributions. }
$$

Moreover,

$$
\|f\|_{h_{p}} \sim \inf \left(\sum_{k=0}^{\infty}\left|\mu_{k}\right|^{p}\right)^{1 / p}
$$

where the infimum is taken over all decompositions of $f$ of the form (2).

Given a (quasi-)Banach space $X$ on $\mathbb{R}^{d}$, a measurable function $f$ belongs to the weighted Wiener amalgam space $W\left(X, \ell_{q}^{v_{s}}\right)\left(\mathbb{R}^{d}\right)(0<q \leq \infty)$ if

$$
\|f\|_{W\left(X, \ell_{q}^{v_{s}}\right)}:=\left(\sum_{k \in \mathbb{Z}^{d}}\left\|\left.f\right|_{[k, k+1)}\right\|_{X}^{q} v_{s}(k)^{q}\right)^{1 / q}<\infty
$$

with the obvious modification for $q=\infty$, where $k+1:=\left(k_{1}+1, \ldots, k_{d}+1\right)$ and $v_{s}(\omega):=(1+|\omega|)^{s}\left(\omega \in \mathbb{R}^{d}, s \geq 0\right)$. For more about amalgam spaces see e.g. Heil [17]. In the unweighted case, i.e. if $s=0$, we omit simply the notation $v_{s}$ and write $W\left(X, \ell_{q}\right)$. In this paper we will use the Wiener amalgam spaces for $X=L_{p}\left(\mathbb{R}^{d}\right), L_{p, \infty}\left(\mathbb{R}^{d}\right), h_{p}\left(\mathbb{R}^{d}\right)$. The smallest closed subspace of $W\left(L_{\infty}, \ell_{q}\right)\left(\mathbb{R}^{d}\right)$ containing continuous functions is denoted by $W\left(C, \ell_{q}\right)\left(\mathbb{R}^{d}\right)(1 \leq q \leq \infty)$. The space $W\left(C, \ell_{1}\right)\left(\mathbb{R}^{d}\right)$ is called Wiener algebra. It is used quite often in Gabor analysis, because it provides a convenient and general class of windows (see e.g. 
Walnut [26] and Gröchenig [13, 14]). As we have seen in Feichtinger and Weisz $[7,8]$, it plays an important rule in summability theory, too.

Observe that $W\left(L_{p}, \ell_{p}\right)\left(\mathbb{R}^{d}\right)=L_{p}\left(\mathbb{R}^{d}\right)$ and

$$
W\left(L_{\infty}, \ell_{1}\right)\left(\mathbb{R}^{d}\right) \subset L_{p}\left(\mathbb{R}^{d}\right) \subset W\left(L_{1}, \ell_{\infty}\right)\left(\mathbb{R}^{d}\right) \quad(1 \leq p \leq \infty) .
$$

In this paper the constants $C$ and $C_{p}$ may vary from line to line and the constants $C_{p}$ are depending only on $p, \alpha$ and $\beta$.

\section{Gabor frames}

Translation and modulation of a function $f$ are defined, respectively, by

$$
T_{x} f(t):=f(t-x) \quad \text { and } \quad M_{\omega} f(t):=e^{2 \pi \imath \omega \cdot t} f(t) \quad\left(x, \omega \in \mathbb{R}^{d}\right) .
$$

The Fourier transform of $f \in L_{1}\left(\mathbb{R}^{d}\right)$ is

$$
\hat{f}(x):=\int_{\mathbb{R}^{d}} f(t) e^{-2 \pi \imath x \cdot t} d t \quad\left(x \in \mathbb{R}^{d}\right),
$$

where $\imath=\sqrt{-1}$. Let $Q$ and $Q_{\alpha}$ denote the cubes

$$
Q=[0,1)^{d}, \quad Q_{\alpha}=[0, \alpha)^{d} \quad(\alpha>0) .
$$

Given a window $g \in L_{2}\left(\mathbb{R}^{d}\right)$ and $\alpha, \beta>0$ we say that the collection

$$
\mathcal{G}(g, \alpha, \beta):=\left\{M_{\beta n} T_{\alpha k} g: k, n \in \mathbb{Z}^{d}\right\}
$$

is a Gabor frame for $L_{2}\left(\mathbb{R}^{d}\right)$ if there exist constants $A, B>0$ such that

$$
A\|f\|_{2}^{2} \leq \sum_{k, n \in \mathbb{Z}^{d}}\left|\left\langle f, M_{\beta n} T_{\alpha k} g\right\rangle\right|^{2} \leq B\|f\|_{2}^{2}
$$

for all $f \in L_{2}\left(\mathbb{R}^{d}\right)$. In this case there exists a canonical dual window $\gamma \in L_{2}\left(\mathbb{R}^{d}\right)$ such that $\mathcal{G}(\gamma, \alpha, \beta)$ is also a Gabor frame for $L_{2}\left(\mathbb{R}^{d}\right)$ and

$$
f=\sum_{k, n \in \mathbb{Z}^{d}}\left\langle f, M_{\beta n} T_{\alpha k} g\right\rangle M_{\beta n} T_{\alpha k} \gamma=\sum_{k, n \in \mathbb{Z}^{d}}\left\langle f, M_{\beta n} T_{\alpha k} \gamma\right\rangle M_{\beta n} T_{\alpha k} g
$$

for all $f \in L_{2}\left(\mathbb{R}^{d}\right)$. This series converges unconditionally in $L_{2}\left(\mathbb{R}^{d}\right)$ and the $\ell_{2}$ norm of the Gabor coefficients $\left(\left\langle f, M_{\beta n} T_{\alpha k} \gamma\right\rangle\right)$ is an equivalent norm on $L_{2}\left(\mathbb{R}^{d}\right)$. For more details we refer to Daubechies [5, Chapter 3], Gröchenig [13, Chapter 5], Benedetto [1] or Heil and Walnut [18].

Recall that the $n$th Fourier coefficient of a $1 / \beta$ periodic function $h \in L_{1}\left(Q_{1 / \beta}\right)$ is given by

$$
\hat{h}(n):=\beta^{d} \int_{Q_{1 / \beta}} h(t) e^{-2 \pi \imath \beta n \cdot t} d t \quad\left(n \in \mathbb{Z}^{d}\right) .
$$


For $g \in W\left(L_{\infty}, \ell_{1}\right)\left(\mathbb{R}^{d}\right)$ and $f \in W\left(L_{1}, \ell_{\infty}\right)\left(\mathbb{R}^{d}\right)$ define the $1 / \beta$-periodic function $m_{g, k}$ by

$$
m_{g, k}(x):=\beta^{-d} \sum_{n \in \mathbb{Z}^{d}}\left(f \cdot T_{\alpha k} \bar{g}\right)(x-n / \beta) \quad\left(k \in \mathbb{Z}^{d}\right) .
$$

It is proved in Gröchenig, Heil and Okoudjou [15] and Feichtinger and Weisz [9] that $m_{g, k} \in L_{1}\left(Q_{1 / \beta}\right)$ and the sum converges unconditionally in $L_{1}\left(Q_{1 / \beta}\right)$. Moreover, $\hat{m}_{g, k}(n)=\left\langle f, M_{\beta n} T_{\alpha k} g\right\rangle$ for all $n \in \mathbb{Z}^{d}$. Gröchenig, Heil and Okoudjou $[14,15]$ (see also Feichtinger and Weisz [9]) verified a new version of Walnut's representation:

$$
R_{g, \gamma} f:=\sum_{k \in \mathbb{Z}^{d}} m_{g, k} T_{\alpha k} \gamma
$$

converges unconditionally in the weak*-topology of $W\left(L_{1}, \ell_{\infty}\right)\left(\mathbb{R}^{d}\right)$ and $R_{g, \gamma}$ is bounded on $W\left(L_{1}, \ell_{\infty}\right)\left(\mathbb{R}^{d}\right)$, i.e.

$$
\left\|R_{g, \gamma} f\right\|_{W\left(L_{1}, \ell_{\infty}\right)} \leq C\|g\|_{W\left(L_{\infty}, \ell_{1}\right)}\|\gamma\|_{W\left(L_{\infty}, \ell_{1}\right)}\|f\|_{W\left(L_{1}, \ell_{\infty}\right)} .
$$

Moreover, if $\mathcal{G}(g, \alpha, \beta)$ is a Gabor frame for $L_{2}\left(\mathbb{R}^{d}\right)$ with dual frame $\mathcal{G}(\gamma, \alpha, \beta)$ then $R_{g, \gamma} f=f$ for all $f \in W\left(L_{1}, \ell_{\infty}\right)\left(\mathbb{R}^{d}\right)$.

\section{The summability kernel}

In this paper we consider the second type of $\theta$-summation, where $\theta$ is given by the product of $d$ one-dimensional functions, $\theta(x):=\theta_{1}\left(x_{1}\right) \cdots \theta_{d}\left(x_{d}\right)$. For $\theta_{i} \in$ $W\left(C, \ell_{1}\right)(\mathbb{R})(i=1, \ldots, d)$ the $N \operatorname{th} \theta$-mean of the Fourier series of $h \in L_{1}\left(Q_{1 / \beta}\right)$ is defined by

$$
\sigma_{N}^{\theta} h(x):=\sum_{n \in \mathbb{Z}^{d}} \prod_{i=1}^{d} \theta_{i}\left(\frac{-n_{i}}{N_{i}}\right) \hat{h}(n) e^{2 \pi \imath \beta n \cdot x} \quad\left(N \in \mathbb{N}_{+}^{d}\right) .
$$

If for each $i \theta_{i}=\mathbf{1}_{(-1,1)}$ then we get the partial sums, if $\theta_{i}(t)=\max (0,1-|t|)$ $(t \in \mathbb{R})$ then the Fejér means.

In this paper we investigate the $\theta$-means and the convergence over a cone

$$
\mathbb{N}_{\tau}^{d}:=\left\{N \in \mathbb{N}^{d}, 2^{-\tau} \leq N_{k} / N_{j} \leq 2^{\tau} ; k, j=1, \ldots, d\right\},
$$

where $\tau \geq 0$ is fixed. Marcinkiewicz and Zygmund [21] proved that the Fejér means $\sigma_{N}^{\theta} h$ of a function $h \in L_{1}\left(\mathbb{T}^{d}\right)$ converge a.e. to $f$ as $N \rightarrow \infty$ provided that $N \in \mathbb{N}_{\tau}^{d}$. Moreover, the (restricted) maximal operator

$$
\sigma_{*}^{\theta} h:=\sup _{N \in \mathbb{N}_{\tau}^{d}}\left|\sigma_{N}^{\theta} h\right|
$$

is bounded from $H_{p}\left(\mathbb{T}^{d}\right)$ to $L_{p}\left(\mathbb{T}^{d}\right)$ whenever $d /(d+1)<p<\infty$ (see Weisz [27]). 
These results are generalized for Gabor series as follows. The $\theta$-means are defined for Gabor series by

$$
\sigma_{g, \gamma, K, N}^{\theta} f:=\sigma_{K, N}^{\theta} f:=\sum_{j=1}^{d} \sum_{k_{j}=-K_{j}}^{K_{j}} \sum_{n \in \mathbb{Z}^{d}} \prod_{i=1}^{d} \theta_{i}\left(\frac{-n_{i}}{N_{i}}\right)\left\langle f, M_{\beta n} T_{\alpha k} g\right\rangle M_{\beta n} T_{\alpha k} \gamma,
$$

respectively, where $K \in \mathbb{N}^{d}, N \in \mathbb{N}_{+}^{d}, g, \gamma \in W\left(L_{\infty}, \ell_{1}\right)\left(\mathbb{R}^{d}\right), f \in W\left(L_{1}, \ell_{\infty}\right)\left(\mathbb{R}^{d}\right)$ and $\theta_{i} \in W\left(C, \ell_{1}\right)(\mathbb{R})(i=1, \ldots, d)$. Under $\sum_{j=1}^{d} \sum_{k_{j}=-K_{j}}^{K_{j}}$ we mean the sum $\sum_{k_{1}=-K_{1}}^{K_{1}} \cdots \sum_{k_{d}=-K_{d}}^{K_{d}}$. Note that the results in [30, 29] cannot be applied to these means. The summation method just defined is not symmetric. It is taken in the frequency domain, only. As we can see later we obtain results for the partial sums in the time domain and for the summability means in the frequency domain, so it is not necessary to consider summability in the time domain, because we verify "better" results. However, we can define a symmetric summability method, see the remark after Corollary 2 .

We will assume that $g, \gamma \in W\left(L_{\infty}, \ell_{1}\right)\left(\mathbb{R}^{d}\right)$ and $\gamma$ is a dual window to $g$. Khristal and Okoudjou [19] proved that in case $g \in W\left(L_{\infty}, \ell_{1}\right)\left(\mathbb{R}^{d}\right)$, the canonical dual $\gamma$ is also in this space. The same result is known for Feichtinger's algebra $\mathbf{S}_{0}\left(\mathbb{R}^{d}\right)$ (see Gröchenig and Leinert [16]).

If $g, \gamma \in W\left(L_{\infty}, \ell_{1}\right)\left(\mathbb{R}^{d}\right)$ such that $\mathcal{G}(g, \alpha, \beta)$ is a Gabor frame for $L_{2}\left(\mathbb{R}^{d}\right)$ with dual frame $\mathcal{G}(\gamma, \alpha, \beta)$ and $\hat{\theta} \in L_{1}\left(\mathbb{R}^{d}\right)$ then

$$
\lim _{K, N \rightarrow \infty} \sigma_{K, N}^{\theta} f=\theta(0) f \quad \text { in } W\left(L_{1}, \ell_{q}\right)\left(\mathbb{R}^{d}\right) \text { norm, } 1 \leq q<\infty
$$

for all $f \in W\left(L_{1}, \ell_{q}\right)\left(\mathbb{R}^{d}\right)$ (see Feichtinger and Weisz [9]). Note that Fejér summation of Gabor series for $L_{1}\left(\mathbb{R}^{d}\right)$ spaces and for special window functions from the Schwartz class was investigated in Grafakos and Lennard [12], too.

Here we investigate a more general maximal $\theta$-operator than in [29]. There we have taken the supremum over the diagonal, i.e., if $K_{1}=\ldots=K_{d}$ and $N_{1}=\ldots=N_{d}$. Similarly to (5), here we define the (restricted) maximal $\theta$ operator on a larger set, namely on a cone $\mathbb{N}_{\tau}^{d}$, by

$$
\sigma_{g, \gamma, *}^{\theta}:=\sigma_{*}^{\theta} f:=\sup _{K \in \mathbb{N}^{d}, N \in \mathbb{N}_{\tau}^{d}}\left|\sigma_{K, N}^{\theta} f\right| .
$$

Recall that $\mathbb{N}_{\tau}^{d}$ was defined in (4). The following result can be proved similarly to [29].

Theorem 2 If $\theta \in W\left(C, \ell_{1}\right)\left(\mathbb{R}^{d}\right), \hat{\theta} \in L_{1}\left(\mathbb{R}^{d}\right)$ and $g, \gamma \in W\left(L_{\infty}, \ell_{1}\right)\left(\mathbb{R}^{d}\right)$ then

$$
\sigma_{K, N}^{\theta} f=\int_{\mathbb{R}^{d}} f(t) F_{K, N}^{\theta}(x, t) d t \quad\left(f \in W\left(L_{1}, \ell_{\infty}\right)\left(\mathbb{R}^{d}\right)\right)
$$


for all $K, N \in \mathbb{N}^{d}$, where the $\theta$-kernels are given by

$$
F_{K, N}^{\theta}(x, t)=\sum_{j=1}^{d} \sum_{k_{j}=-K_{j}}^{K_{j}} \sum_{n \in \mathbb{Z}^{d}}\left(\prod_{i=1}^{d} N_{i} \hat{\theta}_{i}\left(N_{i}\left(\beta\left(x_{i}-t_{i}+n_{i} / \beta\right)\right)\right) \overline{T_{\alpha k} g(t)} T_{\alpha k} \gamma(x) .\right.
$$

Moreover,

$$
\left\|\sigma_{*}^{\theta} f\right\|_{\infty} \leq C\|\hat{\theta}\|_{1}\|g\|_{W\left(L_{\infty}, \ell_{1}\right)}\|\gamma\|_{W\left(L_{\infty}, \ell_{1}\right)}\|f\|_{\infty} .
$$

\section{Summability in Hardy spaces}

In this section we generalize the preceding results and prove that $\sigma_{*}^{\theta}$ is bounded from $h_{p}\left(\mathbb{R}^{d}\right)$ to $L_{p}\left(\mathbb{R}^{d}\right)$ and from $W\left(h_{p}, \ell_{\infty}\right)\left(\mathbb{R}^{d}\right)$ to $W\left(L_{p}, \ell_{\infty}\right)\left(\mathbb{R}^{d}\right)$. As a consequence we obtain the analogue of Marcinkiewicz-Zygmund result for Gabor series, namely, the a.e. convergence of the $\theta$-means for functions from $W\left(L_{1}, \ell_{\infty}\right)$ $\left(\mathbb{R}^{d}\right)$. Observe that $W\left(L_{\infty}, \ell_{p}\right)\left(\mathbb{R}^{d}\right) \subset W\left(L_{\infty}, \ell_{1}\right)\left(\mathbb{R}^{d}\right)$ for $0<p<1$.

Theorem 3 For $\theta_{i} \in W\left(C, \ell_{1}\right)(\mathbb{R})$ assume that $\hat{\theta}_{i}(i=1, \ldots, d)$ is differentiable, $\hat{\theta}_{i}^{\prime}$ is bounded and there exists $1<\delta_{i} \leq 2$ such that

$$
\left|\hat{\theta}_{i}(x)\right|,\left|\hat{\theta}_{i}^{\prime}(x)\right| \leq C|x|^{-\delta_{i}} \quad(x \neq 0) .
$$

Let $p_{0}:=\max \left\{d /(d+1), 1 / \delta_{i}, i=1, \ldots, d\right\}$ and $p_{0}<p \leq 1, \gamma \in W\left(L_{\infty}, \ell_{p}\right)$ $\left(\mathbb{R}^{d}\right)$ and $g \in W\left(L_{\infty}, \ell_{p}\right)\left(\mathbb{R}^{d}\right)$ be $d$ times differentiable such that $\partial_{1}^{i_{1}} \ldots \partial_{d}^{i_{d}} g \in$ $W\left(L_{\infty}, \ell_{p}\right)\left(\mathbb{R}^{d}\right)$ for $i_{1}+\ldots+i_{d}=d$. Then

$$
\left\|\sigma_{*}^{\theta} f\right\|_{p} \leq C_{p, \theta, g}\|\gamma\|_{W\left(L_{\infty}, \ell_{p}\right)}\|f\|_{h_{p}} \quad\left(f \in h_{p}\left(\mathbb{R}^{d}\right)\right) .
$$

Moreover, for all $1<r<\infty$,

$$
\left\|\sigma_{*}^{\theta} f\right\|_{r} \leq C_{1, \theta, g}\|\gamma\|_{W\left(L_{\infty}, \ell_{1}\right)}\|f\|_{h_{r}} \quad\left(f \in h_{r}\left(\mathbb{R}^{d}\right)\right) .
$$

If $p<1$ then

$$
\left\|\sigma_{*}^{\theta} f\right\|_{L_{1, \infty}} \leq C_{p, \theta, g}\|\gamma\|_{W\left(L_{\infty}, \ell_{p}\right)}\|f\|_{1} \quad\left(f \in L_{1}\left(\mathbb{R}^{d}\right)\right) .
$$

Remark 1 Note that

$$
C_{p, \theta, g}:=C_{p} \prod_{j=1}^{d}\left(1+\left\|\hat{\theta}_{j}\right\|_{1}\right)\left(\sum_{i_{1}+\ldots+i_{d}=0, d}\left\|\partial_{1}^{i_{1}} \ldots \partial_{d}^{i_{d}} g\right\|_{W\left(L_{\infty}, \ell_{p}\right)}\right),
$$

where $C_{p}$ is depending only on $p$. Since the constant $C\|\hat{\theta}\|_{1}\|g\|_{W\left(L_{\infty}, \ell_{1}\right)}$ in (7) can also be estimated by $C_{p, \theta, g}$, the same constant appears in (10) and (11) by interpolation. 
Observe that $\hat{\theta}_{i}^{\prime}$ is bounded if $\theta_{i} \in W\left(C, \ell_{1}^{v_{1}}\right)(\mathbb{R})$ (or if $\theta_{i}$ has bounded support). Indeed, in this case $x \theta_{i}(x) \in L_{1}(\mathbb{R})$, which implies the boundedness of $\hat{\theta}_{i}^{\prime}$ $(i=1,2)$.

The next corollary follows from (11) and the usual density argument due to Marcinkiewicz and Zygmund [21].

Corollary 1 If $p<1$ in Theorem 3 then

$$
\lim _{K, N \rightarrow \infty, N \in \mathbb{N}_{\tau}^{d}} \sigma_{K, N}^{\theta} f=\theta(0) R_{g, \gamma} f \quad \text { a.e. }
$$

for all $f \in L_{1}\left(\mathbb{R}^{d}\right)$. If $\mathcal{G}(g, \alpha, \beta)$ is a Gabor frame for $L_{2}\left(\mathbb{R}^{d}\right)$ with dual frame $\mathcal{G}(\gamma, \alpha, \beta)$ then $\sigma_{K, N}^{\theta} f$ converges to $\theta(0) f$ a.e. for all $f \in L_{1}\left(\mathbb{R}^{d}\right)$.

Now we extend the previous theorem to amalgam Hardy spaces.

Theorem 4 Under the conditions of Theorem 3

$$
\left\|\sigma_{*}^{\theta} f\right\|_{W\left(L_{p}, \ell_{\infty}\right)} \leq C_{p, \theta, g}\|\gamma\|_{W\left(L_{\infty}, \ell_{p}\right)}\|f\|_{W\left(h_{p}, \ell_{\infty}\right)} \quad\left(f \in W\left(h_{p}, \ell_{\infty}\right)\left(\mathbb{R}^{d}\right)\right) .
$$

Moreover, for all $1<r<\infty$,

$$
\left\|\sigma_{*}^{\theta} f\right\|_{W\left(L_{r}, \ell_{\infty}\right)} \leq C_{1, \theta, g}\|\gamma\|_{W\left(L_{\infty}, \ell_{1}\right)}\|f\|_{W\left(h_{r}, \ell_{\infty}\right)} \quad\left(f \in W\left(h_{r}, \ell_{\infty}\right)\left(\mathbb{R}^{d}\right)\right) .
$$

If $p<1$ then

$$
\left\|\sigma_{*}^{\theta} f\right\|_{W\left(L_{1, \infty}, \ell_{\infty}\right)} \leq C_{p, \theta, g}\|\gamma\|_{W\left(L_{\infty}, \ell_{p}\right)}\|f\|_{W\left(L_{1}, \ell_{\infty}\right)} \quad\left(f \in W\left(L_{1}, \ell_{\infty}\right)\left(\mathbb{R}^{d}\right)\right) .
$$

Note that $C_{p, \theta, g}$ was defined in Remark 1.

By this theorem we can verify the next extension of Corollary 1. Recall that $W\left(L_{1}, \ell_{\infty}\right)\left(\mathbb{R}^{d}\right)$ is a much larger space than $L_{1}\left(\mathbb{R}^{d}\right)$, in fact $W\left(L_{1}, \ell_{\infty}\right)\left(\mathbb{R}^{d}\right) \supset$ $L_{p}\left(\mathbb{R}^{d}\right)$ for all $1 \leq p \leq \infty$.

Corollary 2 If $p<1$ in Theorem 4 then

$$
\lim _{K, N \rightarrow \infty, N \in \mathbb{N}_{\tau}^{d}} \sigma_{K, N}^{\theta} f=\theta(0) R_{g, \gamma} f \quad \text { a.e. }
$$

for all $f \in W\left(L_{1}, \ell_{\infty}\right)\left(\mathbb{R}^{d}\right)$. If $\mathcal{G}(g, \alpha, \beta)$ is a Gabor frame for $L_{2}\left(\mathbb{R}^{d}\right)$ with dual frame $\mathcal{G}(\gamma, \alpha, \beta)$ then $\sigma_{K, N}^{\theta} f$ converges to $\theta(0) f$ a.e. for all $f \in W\left(L_{1}, \ell_{\infty}\right)\left(\mathbb{R}^{d}\right)$.

Finally we remark that a symmetric summation method in the time and frequency domain can be defined by

$$
\sigma_{K, N}^{\eta, \theta} f:=\sum_{k \in \mathbb{Z}^{d}} \sum_{n \in \mathbb{Z}^{d}} \prod_{i=1}^{d} \eta_{i}\left(\frac{-n_{i}}{N_{i}}\right) \prod_{i=1}^{d} \theta_{i}\left(\frac{-n_{i}}{N_{i}}\right)\left\langle f, M_{\beta n} T_{\alpha k} g\right\rangle M_{\beta n} T_{\alpha k} \gamma,
$$


where $g, \gamma \in W\left(L_{\infty}, \ell_{1}\right)\left(\mathbb{R}^{d}\right), f \in W\left(L_{1}, \ell_{\infty}\right)\left(\mathbb{R}^{d}\right)$ and $\eta, \theta \in W\left(C, \ell_{1}\right)\left(\mathbb{R}^{d}\right)$ with $\eta(0)=1$. If $\eta_{i}=\mathbf{1}_{(-1,1)}$ then $\sigma_{K, N}^{\eta, \theta} f=\sigma_{K, N}^{\theta} f$. If

$$
\sigma_{*}^{\eta, \theta} f:=\sup _{K \in \mathbb{N}^{d}, N \in \mathbb{N}_{\tau}^{d}}\left|\sigma_{K, N}^{\eta, \theta} f\right|
$$

then all the above results can be shown for $\sigma_{K, N}^{\eta, \theta} f$ and $\sigma_{*}^{\eta, \theta} f$ in the same way.

\section{Some summability methods}

After some computation we can see that the next summability methods satisfy the conditions of Theorems 3 and 4 .

Example 1 (Fejér summation) If

$$
\theta(x):=\left\{\begin{array}{ll}
1-|x| & \text { if }|x| \leq 1 \\
0 & \text { if }|x|>1
\end{array} \quad(x \in \mathbb{R})\right.
$$

then $\hat{\theta}(x)=\left(\frac{\sin x / 2}{x / 2}\right)^{2}(x \neq 0)$.

Example 2 (de La Vallée-Poussin summation) Let

$$
\theta(x)=\left\{\begin{array}{ll}
1 & \text { if }|x| \leq 1 / 2 \\
-2|x|+2 & \text { if } 1 / 2<|x| \leq 1 \\
0 & \text { if }|x|>1
\end{array} \quad(x \in \mathbb{R}) .\right.
$$

Example 3 (Jackson-de La Vallée-Poussin summation) Let

$$
\theta(x)=\left\{\begin{array}{ll}
1-3 x^{2} / 2+3|x|^{3} / 4 & \text { if }|x| \leq 1 \\
(2-|x|)^{3} / 4 & \text { if } 1<|x| \leq 2 \\
0 & \text { if }|x|>2
\end{array} \quad(x \in \mathbb{R}) .\right.
$$

The next example generalizes Examples 1, 2 and 3.

Example 4 Let $0=\alpha_{0}<\alpha_{1}<\ldots<\alpha_{m}$ and $\beta_{0}, \ldots, \beta_{m}(m \in \mathbb{N})$ be real numbers, $\beta_{0}=1, \beta_{m}=0$. Assume that $\theta$ is even, $\theta\left(\alpha_{j}\right)=\beta_{j}(j=0,1, \ldots, m)$, $\theta(x)=0$ for $x \geq \alpha_{m}, \theta_{9}$ is a polynomial on the interval $\left[\alpha_{j-1}, \alpha_{j}\right](j=1, \ldots, m)$.

Example 5 (Rogosinski summation) Let

$$
\theta(x)=\left\{\begin{array}{ll}
\cos \pi x / 2 & \text { if }|x| \leq 1+2 j \\
0 & \text { if }|x|>1+2 j
\end{array} \quad(j \in \mathbb{N})\right.
$$

Example 6 (Weierstrass summation) $\theta(x)=e^{-|x|^{\gamma}}(x \in \mathbb{R}, 1 \leq \gamma<\infty)$. If $\gamma=1$ then it is called Abel summation. 
Example $7 \theta(x)=e^{-\left(1+|x|^{q}\right)^{\gamma}}(x \in \mathbb{R}, 1 \leq q<\infty, 0<\gamma<\infty)$.

Example 8 (Picard and Bessel summations) $\theta(x)=\left(1+|x|^{\gamma}\right)^{-\alpha}(x \in$ $\mathbb{R}, 0<\alpha<\infty, 1 \leq \gamma<\infty, \alpha \gamma>2)$.

Example 9 Let

$$
\theta(x):=\left\{\begin{array}{ll}
1 & \text { if }|x| \leq 1 \\
|x|^{-\alpha} & \text { if }|x|>1
\end{array} \quad(x \in \mathbb{R}, 2<\alpha<\infty) .\right.
$$

Example 10 Let

$$
\theta(x):=\left\{\begin{array}{ll}
1 & \text { if } x=0 \\
\frac{1-e^{-|x|^{\alpha}}}{|x|^{\alpha}} & \text { if }|x|>0
\end{array} \quad(x \in \mathbb{R}, 2<\alpha<\infty) .\right.
$$

Example 11 (Riemann summation) If $\theta(x)=\left(\frac{\sin x / 2}{x / 2}\right)^{2}$ then $\hat{\theta}=\max (0,1-$ $|x|)$.

Example 12 (Riesz summation) Let

$$
\theta(x):=\left\{\begin{array}{ll}
\left(1-|x|^{\gamma}\right)^{\alpha} & \text { if }|x| \leq 1 \\
0 & \text { if }|x|>1
\end{array} \quad(x \in \mathbb{R})\right.
$$

for some $0<\alpha<\infty, 1 \leq \gamma<\infty$. We have proved in [27] that

$$
|\hat{\theta}(x)|,\left|\hat{\theta}^{\prime}(x)\right| \leq \frac{C}{|x|^{(\alpha \wedge 1)+1}} .
$$

\section{$7 \quad$ Proof of Theorem 3}

Observe that the integrability of $\hat{\theta}_{i}$ follows from (8). For simplicity we prove the result for $d=2$, only. For $d>2$ the verification is very similar. Assume that $p_{0}<p \leq 1$ and $a$ is an arbitrary $h_{p}(\mathbb{R})$-atom with support $I$, where $I$ is a cube $I=I_{1} \times I_{2}$ and $2^{-L-1}<\left|I_{1}\right|=\left|I_{2}\right| \leq 2^{-L}$ for some $L \in \mathbb{Z}$. Let $4 I_{1}$ denote the interval with the same center as $I_{1}$ and with lenght $4\left|I_{1}\right|$ and $4 I:=4 I_{1} \times 4 I_{2}$.

Step 1. First assume that $a$ is a type 2 atom, i.e., $L \leq 0$. Then

$$
\begin{aligned}
& \int_{\mathbb{R}^{2}}\left|\sigma_{*}^{\theta} a(x)\right|^{p} d x \\
& \leq \quad C_{p} 2^{2 L} \int_{\mathbb{R}^{d}} \sup _{K \in \mathbb{N}^{2}, N \in \mathbb{N}_{\tau}^{2}}\left(\sum_{\left|k_{1}\right| \leq K_{1}} \sum_{\left|k_{2}\right| \leq K_{2}} \sum_{n \in \mathbb{Z}^{2}} \int_{I} N_{1}\left|\hat{\theta}_{1}\left(N_{1} \beta\left(x_{1}-t_{1}+n_{1} / \beta\right)\right)\right|\right. \\
&\left.N_{2}\left|\hat{\theta}_{2}\left(N_{2} \beta\left(x_{2}-t_{2}+n_{2} / \beta\right)\right)\right|\left|T_{\alpha k} g(t)\right|\left|T_{\alpha k} \gamma(x)\right| d t\right)^{p} d x \\
& \leq C_{p} 2^{2 L} \int_{\mathbb{R}^{d}} \sup _{N \in \mathbb{N}_{\tau}^{2}}\left(\sum_{k \in \mathbb{Z}^{2}} \sum_{n \in \mathbb{Z}^{2}} \sum_{l \in \mathcal{L}} \int_{T_{l / \beta} Q_{1 / \beta}} N_{1}\left|\hat{\theta}_{1}\left(N_{1} \beta\left(x_{1}-t_{1}+n_{1} / \beta\right)\right)\right|\right. \\
&\left.N_{2}\left|\hat{\theta}_{2}\left(N_{2} \beta\left(x_{2}-t_{2}+n_{2} / \beta\right)\right)\right|\left|T_{\alpha k} g(t)\right|\left|T_{\alpha k} \gamma(x)\right| d t\right)^{p} d x,
\end{aligned}
$$


where $\mathcal{L} \subset \mathbb{Z}^{2}$ is the smallest finite set, such that $\cup_{l \in \mathcal{L}} T_{l / \beta} Q_{1 / \beta} \supset I$. It is easy to see that $|\mathcal{L}| \sim|I|$. Since

$$
\int_{\mathbb{R}^{2}}|\gamma(x)|^{p} d x \leq\|\gamma\|_{W\left(L_{\infty}, \ell_{p}\right)}^{p}
$$

and

$$
\sum_{n \in \mathbb{Z}^{2}} \int_{T_{l / \beta} Q_{1 / \beta}} N_{1}\left|\hat{\theta}_{1}\left(N_{1} \beta\left(x_{1}-t_{1}+n_{1} / \beta\right)\right)\right| N_{2}\left|\hat{\theta}_{2}\left(N_{2} \beta\left(x_{2}-t_{2}+n_{2} / \beta\right)\right)\right| d t=\left\|\hat{\theta}_{1}\right\|_{1}\left\|\hat{\theta}_{1}\right\|_{2}
$$

for all $N$, we obtain

$$
\begin{aligned}
\int_{\mathbb{R}^{2}}\left|\sigma_{*}^{\theta} a(x)\right|^{p} d x & \leq C_{p} 2^{2 L} \int_{\mathbb{R}^{2}} \sum_{k \in \mathbb{Z}^{2}} \sum_{l \in \mathcal{L}}\left\|\hat{\theta}_{1}\right\|_{1}^{p}\left\|\hat{\theta}_{1}\right\|_{2}^{p} \sup _{T_{l / \beta} Q_{1 / \beta}}\left|T_{\alpha k} g(t)\right|^{p}\left|T_{\alpha k} \gamma(x)\right|^{p} d x \\
& \leq C_{p}\left\|\hat{\theta}_{1}\right\|_{1}^{p}\left\|\hat{\theta}_{1}\right\|_{2}^{p} 2^{2 L} \sum_{k \in \mathbb{Z}^{2}} \sum_{l \in \mathcal{L}} \sup _{T_{l / \beta} Q_{1 / \beta}}\left|T_{\alpha k} g(t)\right|^{p} \int_{\mathbb{R}^{2}}|\gamma(x)|^{p} d x \\
& \leq C_{p}\left\|\hat{\theta}_{1}\right\|_{1}^{p}\left\|\hat{\theta}_{1}\right\|_{2}^{p} 2^{2 L} \sum_{l \in \mathcal{L}}\|g\|_{W\left(L_{\infty}, \ell_{p}\right)}^{p}\|\gamma\|_{W\left(L_{\infty}, \ell_{p}\right)}^{p} \\
& \leq C_{p}\left\|\hat{\theta}_{1}\right\|_{1}^{p}\left\|\hat{\theta}_{1}\right\|_{2}^{p}\|g\|_{W\left(L_{\infty}, \ell_{p}\right)}^{p}\|\gamma\|_{W\left(L_{\infty}, \ell_{p}\right)}^{p} .
\end{aligned}
$$

Next assume that $a$ is a type 1 atom, i.e. $L \geq 0$. Then

$$
\begin{aligned}
& \int_{\mathbb{R}^{2}}\left|\sigma_{*}^{\theta} a(x)\right|^{p} d x \\
\leq & \sum_{n \in \mathbb{Z}^{2}} \int_{\mathbb{R}^{2}} \sup _{K \in \mathbb{N}^{2}, N \in \mathbb{N}_{\tau}^{2}} \mid \int_{I} a(t) \sum_{\left|k_{1}\right| \leq K_{1}\left|k_{2}\right| \leq K_{2}} \sum_{1} \hat{\theta}_{1}\left(N_{1} \beta\left(x_{1}-t_{1}+n_{1} / \beta\right)\right) \\
& N_{2} \hat{\theta}_{2}\left(N_{2} \beta\left(x_{2}-t_{2}+n_{2} / \beta\right)\right) \overline{T_{\alpha k} g(t)} T_{\alpha k} \gamma(x) \\
& \left(\mathbf{1}_{\left\{4 I_{1}-n_{1} / \beta\right\}}\left(x_{1}\right)+\mathbf{1}_{\left\{\left(4 I_{1}-n_{1} / \beta\right)^{c}\right\}}\left(x_{1}\right)\right) \\
& \left.\left(\mathbf{1}_{\left\{4 I_{2}-n_{2} / \beta\right\}}\left(x_{2}\right)+\mathbf{1}_{\left\{\left(4 I_{2}-n_{2} / \beta\right)^{c}\right\}}\left(x_{2}\right)\right) d t\right|^{p} d x \\
= & \sum_{n \in \mathbb{Z}^{2}} \int_{\mathbb{R}^{2} K \in \mathbb{N}^{2}, N \in \mathbb{N}_{\tau}^{2}} \mid \int_{I} a(t) \sum_{\left|k_{1}\right| \leq K_{1}\left|k_{2}\right| \leq K_{2}} N_{1} \hat{\theta}_{1}\left(N_{1} \beta\left(x_{1}-t_{1}\right)\right) \\
& N_{2} \hat{\theta}_{2}\left(N_{2} \beta\left(x_{2}-t_{2}\right)\right) \bar{T}_{\alpha k} g(t) T_{\alpha k} \gamma(x-n / \beta) \\
& \left.\left(\mathbf{1}_{\{4 I\}}(x)+\mathbf{1}_{\left\{\left(4 I_{1}\right)^{c} \times 4 I_{2}\right\}}(x)+\mathbf{1}_{\left\{4 I_{1} \times\left(4 I_{2}\right)^{c}\right\}}(x)+\mathbf{1}_{\left\{\left(4 I_{1}\right)^{c} \times\left(4 I_{2}\right)^{c}\right\}}(x)\right) d t\right|^{p} d x \\
= & \sum_{n \in \mathbb{Z}^{2}}\left(\int_{4 I}+\int_{\left(4 I_{1}\right)^{c} \times 4 I_{2}}+\int_{4 I_{1} \times\left(4 I_{2}\right)^{c}}+\int_{\left(4 I_{1}\right)^{c} \times\left(4 I_{2}\right)^{c}}\right) \\
& \sup _{K \in \mathbb{N}^{2}, N \in \mathbb{N}_{\tau}^{2}}\left|A_{K, N, n}(x)\right|^{p} d x .
\end{aligned}
$$


Step 2. We obtain for the first expression that

$$
\begin{aligned}
\left|A_{K, N, n}(x)\right|= & \mid \int_{I} a(t) \sum_{\left|k_{1}\right| \leq K_{1}} \sum_{\left|k_{2}\right| \leq K_{2}} N_{1} \hat{\theta}_{1}\left(N_{1} \beta\left(x_{1}-t_{1}\right)\right) \\
& N_{2} \hat{\theta}_{2}\left(N_{2} \beta\left(x_{2}-t_{2}\right)\right) \overline{T_{\alpha k} g(t)} T_{\alpha k} \gamma(x-n / \beta) d t \mid \\
\leq & C_{p} 2^{2 L / p} \sum_{k \in \mathbb{Z}^{2}}\left\|\hat{\theta}_{1}\right\|_{1}\left\|\hat{\theta}_{2}\right\|_{1} \sup _{I}\left|T_{\alpha k} g \| T_{\alpha k} \gamma(x-n / \beta)\right| .
\end{aligned}
$$

Since

$$
\sum_{n \in \mathbb{Z}^{2}}\left|T_{\alpha k} \gamma(x-n / \beta)\right|^{p} \leq C_{p}\|\gamma\|_{W\left(L_{\infty}, \ell_{p}\right)}^{p} \quad\left(x \in \mathbb{R}^{2}\right),
$$

we conclude

$$
\begin{aligned}
& \sum_{n \in \mathbb{Z}^{2}} \int_{4 I} \sup _{K \in \mathbb{N}^{2}, N \in \mathbb{N}_{\tau}^{2}}\left|A_{K, N, n}(x)\right|^{p} d x \\
\leq & C_{p} 2^{2 L} \sum_{k \in \mathbb{Z}^{2}} \int_{4 I}\left\|\hat{\theta}_{1}\right\|_{1}^{p}\left\|\hat{\theta}_{2}\right\|_{1}^{p} d x \sup _{I}\left|T_{\alpha k} g\right|^{p}\|\gamma\|_{W\left(L_{\infty}, \ell_{p}\right)}^{p} \\
\leq & C_{p}\left\|\hat{\theta}_{1}\right\|_{1}^{p}\left\|\hat{\theta}_{2}\right\|_{1}^{p}\|g\|_{W\left(L_{\infty}, \ell_{p}\right)}^{p}\|\gamma\|_{W\left(L_{\infty}, \ell_{p}\right)}^{p} .
\end{aligned}
$$

For the other expressions in (15) we split the supremum into two parts:

$$
\sup _{K \in \mathbb{N}^{2}, N \in \mathbb{N}_{\tau}^{2}}\left|A_{K, N, n}(x)\right| \leq \sup _{K \in \mathbb{N}^{2}, N_{1}, N_{2} \geq 2^{L-\tau}}\left|A_{K, N, n}(x)\right|+\sup _{\substack{K \in \mathbb{N}^{2}, N \in \mathbb{N}_{\tau}^{2} \\ N_{1}, N_{2}<2^{L}}}\left|A_{K, N, n}(x)\right| .
$$

Step 3. Let $x \in\left(4 I_{1}\right)^{c} \times 4 I_{2}$ and $N_{1}, N_{2} \geq 2^{L-\tau}$. Then

$$
\begin{aligned}
& \left|A_{K, N, n}(x)\right| \\
\leq & C_{p} 2^{2 L / p} \sum_{k \in \mathbb{Z}^{2}} \int_{I_{1}} N_{1}\left|\hat{\theta}_{1}\left(N_{1} \beta\left(x_{1}-t_{1}\right)\right)\right| d t_{1} \\
& \int_{I_{2}} N_{2}\left|\hat{\theta}_{2}\left(N_{2} \beta\left(x_{2}-t_{2}\right)\right)\right| d t_{2} \sup _{I}\left|T_{\alpha k} g\right|\left|T_{\alpha k} \gamma(x-n / \beta)\right| \\
\leq & C_{p} 2^{2 L / p} \sum_{k \in \mathbb{Z}^{2}}\left\|\hat{\theta}_{2}\right\|_{1} \int_{I_{1}} N_{1}\left|N_{1} \beta\left(x_{1}-t_{1}\right)\right|^{-\delta_{1}} d t_{1} \sup _{I}\left|T_{\alpha k} g \| T_{\alpha k} \gamma(x-n / \beta)\right| .
\end{aligned}
$$

Let us denote by $B(c, h)(c \in \mathbb{R}, h>0)$ the interval $\{x \in \mathbb{R}:|x-c|<h\}$. If $c=\left(c_{1}, c_{2}\right)$ is the center of $I$ and $x_{1} \in B\left(c_{1},\left(i_{1}+1\right) 2^{-L}\right) \backslash B\left(c_{1}, i_{1} 2^{-L}\right)\left(i_{1} \geq 1\right)$ then

$$
\begin{aligned}
& \left|A_{K, N, n}(x)\right| \\
\leq & C_{p} 2^{2 L / p}\left\|\hat{\theta}_{2}\right\|_{1} N_{1}^{1-\delta_{1}} \int_{I_{1}}\left(i_{1} 2^{-L}\right)^{-\delta_{1}} d t_{1} \sum_{k \in \mathbb{Z}^{2}} \sup _{I}\left|T_{\alpha k} g\right|\left|T_{\alpha k} \gamma(x-n / \beta)\right| \\
\leq & C_{p} 2^{2 L / p}\left\|\hat{\theta}_{2}\right\|_{1} i_{1}^{-\delta_{1}} \sum_{k \in \mathbb{Z}^{2}} \sup _{I}\left|T_{\alpha k} g\right|\left|T_{\alpha k} \gamma(x-n / \beta)\right|
\end{aligned}
$$


because $N_{1} \geq 2^{L-\tau}$. Thus

$$
\begin{aligned}
\sum_{n \in \mathbb{Z}^{2}} & \int_{\left(4 I_{1}\right)^{c} \times 4 I_{2}} \sup _{K \in \mathbb{N}^{2}, N_{1}, N_{2} \geq 2^{L-\tau}}\left|A_{K, N, n}(x)\right|^{p} d x \\
\leq & \sum_{n \in \mathbb{Z}^{2}} \sum_{i_{1}=1}^{\infty} \int_{B\left(c_{1},\left(i_{1}+1\right) 2^{-L}\right) \backslash B\left(c_{1}, i_{1} 2^{-L}\right)} \int_{4 I_{2}} \sup _{K \in \mathbb{N}^{2}, N_{1}, N_{2} \geq 2^{L-\tau}}\left|A_{K, N, n}(x)\right|^{p} d x \\
\leq & C_{p} 2^{2 L}\left\|\hat{\theta}_{2}\right\|_{1}^{p} \sum_{i_{1}=1}^{\infty} i_{1}^{-\delta_{1} p} \int_{B\left(c_{1},\left(i_{1}+1\right) 2^{-L}\right) \backslash B\left(c_{1}, i_{1} 2^{-L}\right)} \\
& \int_{4 I_{2}} \sum_{n \in \mathbb{Z}^{2}} \sum_{k \in \mathbb{Z}^{2}} \sup _{I}\left|T_{\alpha k} g\right|^{p}\left|T_{\alpha k} \gamma(x-n / \beta)\right|^{p} d x \\
\leq & C_{p}\left\|\hat{\theta}_{2}\right\|_{1}^{p}\|g\|_{W\left(L_{\infty}, \ell_{p}\right)}^{p}\|\gamma\|_{W\left(L_{\infty}, \ell_{p}\right)}^{p} \sum_{i_{1}=1}^{\infty} i_{1}^{-\delta_{1} p} \\
\leq & C_{p}\left\|\hat{\theta}_{2}\right\|_{1}^{p}\|g\|_{W\left(L_{\infty}, \ell_{p}\right)}^{p}\|\gamma\|_{W\left(L_{\infty}, \ell_{p}\right)}^{p},
\end{aligned}
$$

if $d / \delta_{1}<p \leq 1$.

Step 4. Let $x \in\left(4 I_{1}\right)^{c} \times 4 I_{2}$ and $N_{1}, N_{2}<2^{L}$. Then

$$
\begin{aligned}
A_{K, N, n}(x)= & \int_{I} a(t) \sum_{\left|k_{1}\right| \leq K_{1}} \sum_{\left|k_{2}\right| \leq K_{2}} N_{1}\left(\hat{\theta}_{1}\left(N_{1} \beta\left(x_{1}-t_{1}\right)\right)-\hat{\theta}_{1}\left(N_{1} \beta\left(x_{1}-c_{1}\right)\right)\right) \\
& +\int_{I} a(t) \sum_{\left|k_{1}\right| \leq K_{1}} \sum_{\left|k_{2}\right| \leq K_{2}} N_{1} \hat{\theta}_{1}\left(N_{1} \beta\left(x_{1}-c_{1}\right)\right) \\
=: & B_{K, N, n}(x)+C_{2, N, n}(x) .
\end{aligned}
$$

By the Lagrange theorem there exists $0<v<1$ such that

$$
\begin{aligned}
& \left|B_{K, N, n}(x)\right| \\
= & \mid \int_{I} a(t) \sum_{\left|k_{1}\right| \leq K_{1}} \sum_{\left|k_{2}\right| \leq K_{2}} N_{1} \hat{\theta}_{1}^{\prime}\left(N_{1} \beta\left(x_{1}-c_{1}\right)-N_{1} \beta v\left(t_{1}-c_{1}\right)\right) \\
& N_{1} \beta\left(t_{1}-c_{1}\right) N_{2} \hat{\theta}_{2}\left(N_{2} \beta\left(x_{2}-t_{2}\right) \overline{T_{\alpha k} g(t)} T_{\alpha k} \gamma(x-n / \beta) d t \mid\right. \\
\leq & C_{p} 2^{2 L / p-L} \sum_{k \in \mathbb{Z}^{2}}\left\|\hat{\theta}_{2}\right\|_{1} \int_{I_{1}} N_{1}^{2}\left|N_{1} \beta\left(x_{1}-c_{1}\right)-N_{1} \beta v\left(t_{1}-c_{1}\right)\right|^{-\delta_{1}} d t_{1} \\
& \sup _{I}\left|T_{\alpha k} g \| T_{\alpha k} \gamma(x-n / \beta)\right| \\
\leq & C_{p} 2^{2 L / p-L}\left\|\hat{\theta}_{2}\right\|_{1} N_{1}^{2-\delta_{1}} \int_{I_{1}}\left(i_{1} 2^{-L}\right)^{-\delta_{1}} d t_{1} \sum_{k \in \mathbb{Z}^{2}} \sup _{I}\left|T_{\alpha k} g \| T_{\alpha k} \gamma(x-n / \beta)\right|
\end{aligned}
$$




$$
\leq C_{p} 2^{2 L / p}\left\|\hat{\theta}_{2}\right\|_{1} i_{1}^{-\delta_{1}} \sum_{k \in \mathbb{Z}^{2}} \sup _{I}\left|T_{\alpha k} g \| T_{\alpha k} \gamma(x-n / \beta)\right|
$$

whenever $N_{1}<2^{L}$ and $x_{1} \in B\left(c_{1},\left(i_{1}+1\right) 2^{-L}\right) \backslash B\left(c_{1}, i_{1} 2^{-L}\right)\left(i_{1} \geq 1\right)$. Thus

$$
\sum_{n \in \mathbb{Z}^{2}} \int_{\left(4 I_{1}\right)^{c} \times 4 I_{2}} \sup _{\substack{K \in \mathbb{N}^{2}, N \in \mathbb{N}_{T}^{2} \\ N_{1}, N_{2}<L^{L}}}\left|B_{K, N, n}(x)\right|^{p} d x \leq C_{p}\left\|\hat{\theta}_{2}\right\|_{1}^{p}\|g\|_{W\left(L_{\infty}, \ell_{p}\right)}^{p}\|\gamma\|_{W\left(L_{\infty}, \ell_{p}\right)}^{p}
$$

follows as in (18).

Since $\hat{\theta}_{2}$ is bounded, we have

$$
\begin{aligned}
& \left|C_{K, N, n}(x)\right| \\
\leq & C_{p} 2^{2 L / p} \sum_{k \in \mathbb{Z}^{2}} \int_{I_{1}} N_{1}\left|\hat{\theta}_{1}\left(N_{1} \beta\left(x_{1}-c_{1}\right)\right)\right| d t_{1} \\
& \int_{I_{2}} N_{2}\left|\hat{\theta}_{2}\left(N_{2} \beta\left(x_{2}-t_{2}\right)\right)\right| d t_{2} \sup _{I}\left|T_{\alpha k} g\right|\left|T_{\alpha k} \gamma(x-n / \beta)\right| \\
\leq & C_{p} 2^{2 L / p-L} \sum_{k \in \mathbb{Z}^{2}} N_{1}^{2-\delta_{1}} \int_{I_{1}}\left|x_{1}-c_{1}\right|^{-\delta_{1}} d t_{1} \sup _{I}\left|T_{\alpha k} g\right|\left|T_{\alpha k} \gamma(x-n / \beta)\right| \\
\leq & C_{p} 2^{2 L / p-L} N_{1}^{2-\delta_{1}} \int_{I_{1}}\left(i_{1} 2^{-L}\right)^{-\delta_{1}} d t_{1} \sum_{k \in \mathbb{Z}^{2}} \sup _{I}\left|T_{\alpha k} g\right|\left|T_{\alpha k} \gamma(x-n / \beta)\right| \\
\leq & C_{p} 2^{2 L / p} i_{1}^{-\delta_{1}} \sum_{k \in \mathbb{Z}^{2}} \sup _{I}\left|T_{\alpha k} g\right|\left|T_{\alpha k} \gamma(x-n / \beta)\right|,
\end{aligned}
$$

whenever $N \in \mathbb{N}_{\tau}^{2}, N_{1}<2^{L}$ and $x_{1} \in B\left(c_{1},\left(i_{1}+1\right) 2^{-L}\right) \backslash B\left(c_{1}, i_{1} 2^{-L}\right)\left(i_{1} \geq 1\right)$ and so

$$
\sum_{n \in \mathbb{Z}^{2}} \int_{\left(4 I_{1}\right)^{c} \times 4 I_{2}} \sup _{\substack{K \in \mathbb{N}^{2}, N \in \mathbb{N}_{T}^{2} \\ N_{1}, N_{2}<2^{L}}}\left|C_{K, N, n}(x)\right|^{p} d x \leq C_{p}\|g\|_{W\left(L_{\infty}, \ell_{p}\right)}^{p}\|\gamma\|_{W\left(L_{\infty}, \ell_{p}\right)}^{p} .
$$

Step 5. The integration over $I_{1} \times\left(4 I_{2}\right)^{c}$ is similar.

Step 6. If $x \in\left(4 I_{1}\right)^{c} \times\left(4 I_{2}\right)^{c}$ and $N_{1}, N_{2} \geq 2^{L-\tau}$ then

$$
\begin{aligned}
\left|A_{K, N, n}(x)\right| \leq & C_{p} 2^{2 L / p} \sum_{k \in \mathbb{Z}^{2}} \int_{I_{1}} N_{1}\left|\hat{\theta}_{1}\left(N_{1} \beta\left(x_{1}-t_{1}\right)\right)\right| d t_{1} \\
& \int_{I_{2}} N_{2}\left|\hat{\theta}_{2}\left(N_{2} \beta\left(x_{2}-t_{2}\right)\right)\right| d t_{2} \sup _{I}\left|T_{\alpha k} g\right|\left|T_{\alpha k} \gamma(x-n / \beta)\right| \\
\leq & C_{p} 2^{2 L / p} \sum_{k \in \mathbb{Z}^{2}} N_{1}^{1-\delta_{1}} \int_{I_{1}}\left|x_{1}-t_{1}\right|^{-\delta_{1}} d t_{1} \\
& N_{2}^{1-\delta_{2}} \int_{I_{2}}\left|x_{2}-t_{2}\right|^{-\delta_{2}} d t_{2} \sup _{I}\left|T_{\alpha k} g \| T_{\alpha k} \gamma(x-n / \beta)\right|
\end{aligned}
$$




$$
\begin{aligned}
\leq & C_{p} 2^{2 L / p+L\left(1-\delta_{1}\right)+L\left(1-\delta_{2}\right)} \int_{I_{1}}\left(i_{1} 2^{-L}\right)^{-\delta_{1}} d t_{1} \int_{I_{2}}\left(i_{2} 2^{-L}\right)^{-\delta_{2}} d t_{2} \\
& \sum_{k \in \mathbb{Z}^{2}} \sup _{I}\left|T_{\alpha k} g\right|\left|T_{\alpha k} \gamma(x-n / \beta)\right| \\
\leq & C_{p} 2^{2 L / p} i_{1}^{-\delta_{1}} i_{2}^{-\delta_{2}} \sum_{k \in \mathbb{Z}^{2}} \sup _{I}\left|T_{\alpha k} g\right|\left|T_{\alpha k} \gamma(x-n / \beta)\right| .
\end{aligned}
$$

Here we have assumed that $x_{1} \in B\left(c_{1},\left(i_{1}+1\right) 2^{-L}\right) \backslash B\left(c_{1}, i_{1} 2^{-L}\right)$ and $x_{2} \in$ $B\left(c_{2},\left(i_{2}+1\right) 2^{-L}\right) \backslash B\left(c_{2}, i_{2} 2^{-L}\right)\left(i_{1}, i_{2} \geq 1\right)$. Hence

$$
\begin{aligned}
\sum_{n \in \mathbb{Z}^{2}} & \int_{\left(4 I_{1}\right)^{c} \times\left(4 I_{2}\right)^{c}} \sup _{K \in \mathbb{N}^{2}, N_{1}, N_{2} \geq 2^{L-\tau}}\left|A_{K, N, n}(x)\right|^{p} d x \\
\leq & C_{p} 2^{2 L} \sum_{i_{1}=1}^{\infty} i_{1}^{-\delta_{1} p} \sum_{i_{2}=1}^{\infty} i_{2}^{-\delta_{2} p} \int_{B\left(c_{1},\left(i_{1}+1\right) 2^{-L}\right) \backslash B\left(c_{1}, i_{1} 2^{-L}\right)} \int_{B\left(c_{2},\left(i_{2}+1\right) 2^{-L}\right) \backslash B\left(c_{2}, i_{2} 2^{-L}\right)} \\
& \sum_{n \in \mathbb{Z}^{2}} \sum_{k \in \mathbb{Z}^{2}} \sup _{I}\left|T_{\alpha k} g\right|^{p}\left|T_{\alpha k} \gamma(x-n / \beta)\right|^{p} d x \\
\leq & C_{p}\|g\|_{W\left(L_{\infty}, \ell_{p}\right)}^{p}\|\gamma\|_{W\left(L_{\infty}, \ell_{p}\right)}^{p} .
\end{aligned}
$$

Step 7. Let $x \in\left(4 I_{1}\right)^{c} \times\left(4 I_{2}\right)^{c}$ and $N_{1}, N_{2}<2^{L}$. We split the terms $B_{K, N, n}$ and $C_{K, N, n}$ into two parts:

$$
\begin{aligned}
& \int_{I} a(t) \sum_{\left|k_{1}\right| \leq K_{1}} \sum_{\left|k_{2}\right| \leq K_{2}} N_{1}\left(\hat{\theta}_{1}\left(N_{1} \beta\left(x_{1}-t_{1}\right)\right)-\hat{\theta}_{1}\left(N_{1} \beta\left(x_{1}-c_{1}\right)\right)\right) \\
& N_{2}\left(\hat{\theta}_{2}\left(N_{2} \beta\left(x_{2}-t_{2}\right)\right)-\hat{\theta}_{2}\left(N_{2} \beta\left(x_{2}-c_{2}\right)\right)\right) \overline{T_{\alpha k} g(t)} T_{\alpha k} \gamma(x-n / \beta) d t \\
& +\int_{I} a(t) \sum_{\left|k_{1}\right| \leq K_{1}} \sum_{\left|k_{2}\right| \leq K_{2}} N_{1}\left(\hat{\theta}_{1}\left(N_{1} \beta\left(x_{1}-t_{1}\right)\right)-\hat{\theta}_{1}\left(N_{1} \beta\left(x_{1}-c_{1}\right)\right)\right) \\
& N_{2} \hat{\theta}_{2}\left(N_{2} \beta\left(x_{2}-c_{2}\right)\right) \overline{T_{\alpha k} g(t)} T_{\alpha k} \gamma(x-n / \beta) d t \\
=: \quad & B_{1, K, N, n}(x)+B_{2, K, N, n}(x) .
\end{aligned}
$$

By the Lagrange mean value theorem,

$$
\begin{array}{rl} 
& \left|B_{1, K, N, n}(x)\right| \\
\mid \int_{I} & a(t) \sum_{\left|k_{1}\right| \leq K_{1}} \sum_{\left|k_{2}\right| \leq K_{2}} N_{1} \hat{\theta}_{1}^{\prime}\left(N_{1} \beta\left(x_{1}-c_{1}\right)-N_{1} \beta v_{1}\left(t_{1}-c_{1}\right)\right) \\
& N_{1} \beta\left(t_{1}-c_{1}\right) N_{2} \hat{\theta}_{2}^{\prime}\left(N_{2} \beta\left(x_{2}-c_{2}\right)-N_{2} \beta v_{2}\left(t_{2}-c_{2}\right)\right) N_{2} \beta\left(t_{2}-c_{2}\right) \\
& \overline{T_{\alpha k} g(t)} T_{\alpha k} \gamma(x-n / \beta) d t \mid
\end{array}
$$




$$
\begin{aligned}
\leq & C_{p} 2^{2 L / p-2 L} \sum_{k \in \mathbb{Z}^{2}} N_{1}^{2-\delta_{1}} N_{2}^{2-\delta_{2}} \int_{I_{1}}\left|\left(x_{1}-c_{1}\right)-v_{1}\left(t_{1}-c_{1}\right)\right|^{-\delta_{1}} d t_{1} \\
& \int_{I_{2}}\left|\left(x_{2}-c_{2}\right)-v_{2}\left(t_{2}-c_{2}\right)\right|^{-\delta_{2}} d t_{2} \sup _{I}\left|T_{\alpha k} g\right|\left|T_{\alpha k} \gamma(x-n / \beta)\right| \\
\leq & C_{p} 2^{2 L / p} i_{1}^{-\delta_{1}} i_{2}^{-\delta_{2}} \sum_{k \in \mathbb{Z}^{2}} \sup _{I}\left|T_{\alpha k} g\right|\left|T_{\alpha k} \gamma(x-n / \beta)\right|,
\end{aligned}
$$

whenever $N_{1}, N_{2}<2^{L}$ and $x_{1} \in B\left(c_{1},\left(i_{1}+1\right) 2^{-L}\right) \backslash B\left(c_{1}, i_{1} 2^{-L}\right), x_{2} \in B\left(c_{2},\left(i_{2}+\right.\right.$ 1) $\left.2^{-L}\right) \backslash B\left(c_{2}, i_{2} 2^{-L}\right)\left(i_{1}, i_{2} \geq 1\right)$. Similarly to (20),

$$
\sum_{n \in \mathbb{Z}^{2}} \int_{\left(4 I_{1}\right)^{c} \times\left(4 I_{2}\right)^{c}} \sup _{\substack{K \in \mathbb{N}^{2}, N \in \mathbb{N}_{\tau}^{2} \\ N_{1}, N_{2}<2^{L}}}\left|B_{1, K, N, n}(x)\right|^{p} d x \leq C_{p}\|g\|_{W\left(L_{\infty}, \ell_{p}\right)}^{p}\|\gamma\|_{W\left(L_{\infty}, \ell_{p}\right)}^{p} .
$$

Since $\hat{\theta}_{i}$ and $\hat{\theta}_{i}^{\prime}$ are bounded,

$$
\left|\hat{\theta}_{i}(x)\right|,\left|\hat{\theta}_{i}^{\prime}(x)\right| \leq C|x|^{-\delta_{i} \eta_{i}} \quad(x \neq 0, i=1,2)
$$

for all fixed $\eta_{i} \in[0,1]$. Applying this we can see that

$$
\begin{aligned}
& \left|B_{2, K, N, n}(x)\right| \\
= & \mid \int_{I} a(t) \sum_{\left|k_{1}\right| \leq K_{1}} \sum_{\left|k_{2}\right| \leq K_{2}} N_{1} \hat{\theta}_{1}^{\prime}\left(N_{1} \beta\left(x_{1}-c_{1}\right)-N_{1} \beta v_{1}\left(t_{1}-c_{1}\right)\right) \\
& N_{1} \beta\left(t_{1}-c_{1}\right) N_{2} \hat{\theta}_{2}\left(N_{2} \beta\left(x_{2}-c_{2}\right) \overline{T_{\alpha k} g(t)} T_{\alpha k} \gamma(x-n / \beta) d t \mid\right. \\
\leq & C_{p} 2^{2 L / p-L} \sum_{k \in \mathbb{Z}^{2}} N_{1}^{2-\delta_{1} \eta_{1}} N_{2}^{1-\delta_{2} \eta_{2}} \int_{I_{1}}\left|\left(x_{1}-c_{1}\right)-v_{1}\left(t_{1}-c_{1}\right)\right|^{-\delta_{1} \eta_{1}} d t_{1} \\
& \int_{I_{2}}\left|x_{2}-c_{2}\right|^{-\delta_{2} \eta_{2}} d t_{2} \sup _{I}\left|T_{\alpha k} g\right|\left|T_{\alpha k} \gamma(x-n / \beta)\right| .
\end{aligned}
$$

Since $N_{1}^{2-\delta_{1} \eta_{1}} N_{2}^{1-\delta_{2} \eta_{2}} \leq C N_{1}^{3 / 2-\delta_{1} \eta_{1}} N_{2}^{3 / 2-\delta_{2} \eta_{2}}$, let us choose $\eta_{i}$ such that $3 / 2-$ $\delta_{i} \eta_{i} \geq 0$, say $\eta_{i}=1 \wedge 3 / 2 \delta_{i},(i=1,2)$. This implies

$$
\left|B_{2, K, N, n}(x)\right| \leq C_{p} 2^{2 L / p} i_{1}^{-\delta_{1} \eta_{1}} i_{2}^{-\delta_{2} \eta_{2}} \sum_{k \in \mathbb{Z}^{2}} \sup _{I}\left|T_{\alpha k} g\right|\left|T_{\alpha k} \gamma(x-n / \beta)\right|,
$$

and

$$
\sum_{n \in \mathbb{Z}^{2}} \int_{\left(4 I_{1}\right)^{c} \times\left(4 I_{2}\right)^{c}} \sup _{\substack{K \in \mathbb{N}^{2}, N \in \mathbb{N}^{2} \\ N_{1}, N_{2}<2^{L}}}\left|B_{2, K, N, n}(x)\right|^{p} d x \leq C_{p}\|g\|_{W\left(L_{\infty}, \ell_{p}\right)}^{p}\|\gamma\|_{W\left(L_{\infty}, \ell_{p}\right)}^{p},
$$

when $\delta_{i} \eta_{i} p>1$, in other words $p>p_{0}$. Similarly, 


$$
\begin{aligned}
& \int_{I} a(t) \sum_{\left|k_{1}\right| \leq K_{1}} \sum_{\left|k_{2}\right| \leq K_{2}} N_{1} \hat{\theta}_{1}\left(N_{1} \beta\left(x_{1}-c_{1}\right)\right) \\
& N_{2}\left(\hat{\theta}_{2}\left(N_{2} \beta\left(x_{2}-t_{2}\right)\right)-\hat{\theta}_{2}\left(N_{2} \beta\left(x_{2}-c_{2}\right)\right)\right) \overline{T_{\alpha k} g(t)} T_{\alpha k} \gamma(x-n / \beta) d t \\
& +\int_{I} a(t) \sum_{\left|k_{1}\right| \leq K_{1}} \sum_{\left|k_{2}\right| \leq K_{2}} N_{1} \hat{\theta}_{1}\left(N_{1} \beta\left(x_{1}-c_{1}\right)\right) \\
& N_{2} \hat{\theta}_{2}\left(N_{2} \beta\left(x_{2}-c_{2}\right)\right) \overline{T_{\alpha k} g(t)} T_{\alpha k} \gamma(x-n / \beta) d t \\
=: & C_{1, K, N, n}(x)+C_{2, K, N, n}(x) .
\end{aligned}
$$

The term $C_{1, K, N, n}$ can be handled as $B_{2, K, N, n}$. For the second term we introduce the $l$ th Taylor polynomial of $T_{\alpha k} g$ by

$$
P_{l} T_{\alpha k} g(t):=\sum_{j=0}^{l} \sum_{i_{1}+i_{2}=j} \partial_{1}^{i_{1}} \partial_{2}^{i_{2}} T_{\alpha k} g(c) \prod_{j=1}^{2} \frac{\left(t_{j}-c_{j}\right)^{i_{j}}}{i_{j} !} .
$$

By condition (iii) of the definition of the atom,

$$
\begin{aligned}
C_{2, K, N, n}(x)= & \int_{I} a(t) \sum_{\left|k_{1}\right| \leq K_{1}} \sum_{\left|k_{2}\right| \leq K_{2}} N_{1} \hat{\theta}_{1}\left(N_{1} \beta\left(x_{1}-c_{1}\right)\right) N_{2} \hat{\theta}_{2}\left(N_{2} \beta\left(x_{2}-c_{2}\right)\right) \\
& \left(\overline{T_{\alpha k} g(t)}-\overline{P_{1} T_{\alpha k} g(t)}\right) T_{\alpha k} \gamma(x-n / \beta) d t .
\end{aligned}
$$

Taylor's formula implies

$$
\begin{aligned}
& \left|C_{2, K, N, n}(x)\right| \\
= & \mid \int_{I} a(t) \sum_{\left|k_{1}\right| \leq K_{1}} \sum_{\left|k_{2}\right| \leq K_{2}} N_{1} \hat{\theta}_{1}\left(N_{1} \beta\left(x_{1}-c_{1}\right)\right) N_{2} \hat{\theta}_{2}\left(N_{2} \beta\left(x_{2}-c_{2}\right)\right) \\
& \sum_{i_{1}+i_{2}=2} \partial_{1}^{i_{1}} \partial_{2}^{i_{2}} \overline{T_{\alpha k} g(c+v(t-c))} \prod_{j=1}^{2} \frac{\overline{\left(t_{j}-c_{j}\right)}}{i_{j} !} T_{\alpha k} \gamma(x-n / \beta) d t \mid \\
\leq & C_{p} 2^{2 L / p-2 L} \sum_{k \in \mathbb{Z}^{2}} N_{1}^{1-\delta_{1}} N_{2}^{1-\delta_{2}} \int_{I_{1}}\left|x_{1}-c_{1}\right|^{-\delta_{1}} d t_{1} \int_{I_{2}}\left|x_{2}-c_{2}\right|^{-\delta_{2}} d t_{2} \\
\leq & \sup _{I}\left|\sum_{i_{1}+i_{2}=2} \partial_{1}^{i_{1}} \partial_{2}^{i_{2}} T_{\alpha k} g\right|\left|T_{\alpha k} \gamma(x-n / \beta)\right| \\
& \sum_{k \in \mathbb{Z}^{2}} \sup _{I}\left|\sum_{i_{1}+i_{2}=2} \partial_{1}^{i_{1}} \partial_{2}^{i_{2}} T_{\alpha k} g\right|\left|T_{\alpha k} \gamma(x-n / \beta)\right| \\
\leq & C_{p} 2^{2 L / p} i_{1}^{-\delta_{1}} i_{2}^{-\delta_{2}} \sum_{k \in \mathbb{Z}^{2}} \sup _{I}\left|\sum_{i_{1}+i_{2}=2} \partial_{1}^{i_{1}} \partial_{2}^{i_{2}} T_{\alpha k} g\right|\left|T_{\alpha k} \gamma(x-n / \beta)\right|,
\end{aligned}
$$


because $1<\delta_{i} \leq 2(i=1,2)$. Hence

$$
\begin{aligned}
& \sum_{n \in \mathbb{Z}^{2}} \int_{\left(4 I_{1}\right)^{c} \times 4 I_{2}} \sup _{\substack{K \in \mathbb{N}^{2}, N \in \mathbb{N}^{2} \\
N_{1}, N_{2}<2^{L}}}\left|C_{2, K, N, n}(x)\right|^{p} d x \\
& \leq C_{p}\left\|\sum_{i_{1}+i_{2}=2} \partial_{1}^{i_{1}} \partial_{2}^{i_{2}} T_{\alpha k} g\right\|_{W\left(L_{\infty}, \ell_{p}\right)}^{p}\|\gamma\|_{W\left(L_{\infty}, \ell_{p}\right)}^{p} .
\end{aligned}
$$

Consequently,

$$
\begin{aligned}
& \int_{\mathbb{R}^{2}}\left|\sigma_{*}^{\theta} a(x)\right|^{p} d x \\
& \quad \leq C_{p}\left(1+\left\|\hat{\theta}_{1}\right\|_{1}^{p}\right)\left(1+\left\|\hat{\theta}_{2}\right\|_{1}^{p}\right)\left(\sum_{i_{1}+i_{2}=0,2}\left\|\partial_{1}^{i_{1}} \partial_{2}^{i_{2}} g\right\|_{W\left(L_{\infty}, \ell_{p}\right)}^{p}\right)\|\gamma\|_{W\left(L_{\infty}, \ell_{p}\right)}^{p},
\end{aligned}
$$

which completes the proof of (9). Inequalities (10) and (11) can be obtained by (7) and by real interpolation in the usual way.

\section{Proof of Theorem 4}

We prove the result again for $d=2$. Obviously,

$$
\int_{m}^{m+1}\left|\sigma_{*}^{\theta} f(x)\right|^{p} d x \leq \sum_{r \in \mathbb{Z}^{2}} \int_{m}^{m+1}\left|\sigma_{*}^{\theta}\left(\left.f\right|_{[r, r+1)}\right)(x)\right|^{p} d x
$$

for each $m \in \mathbb{Z}^{2}$. Let $\left.f\right|_{[r, r+1)}=\sum_{s=0}^{\infty} \mu_{r, s} a_{r, s}$ be an atomic decomposition of $\left.f\right|_{[r, r+1)} \in h_{p}\left(\mathbb{R}^{2}\right)\left(p_{0}<p \leq 1\right)$ such that

$$
\sum_{s=0}^{\infty}\left|\mu_{r, s}\right|^{p} \leq C_{p}\left\|\left.f\right|_{[r, r+1)}\right\|_{h_{p}}^{p} \leq C_{p}\|f\|_{W\left(h_{p}, \ell_{\infty}\right)}^{p} .
$$

Since $\operatorname{supp} \phi \subset[0,1 / 2]^{2}$ we have $\left.\operatorname{supp} f\right|_{[r, r+1)} * \phi_{t} \subset[r, r+3 / 2)$, where $r \in \mathbb{Z}^{2}$, $0<t<1$. So we may assume that $a_{r, s}$ is supported in a cube $I_{r, s} \subset[r, r+3 / 2)$ and $2^{-L_{r, s}-1}<\left|I_{r, s, 1}\right|=\left|I_{r, s, 2}\right| \leq 2^{-L_{r, s}}$ for some $L_{r, s} \in \mathbb{Z}$. Thus

$$
\int_{m}^{m+1}\left|\sigma_{*}^{\theta} f(x)\right|^{p} d x \leq \sum_{r \in \mathbb{Z}^{2}} \sum_{s=0}^{\infty}\left|\mu_{r, s}\right|^{p} \int_{m}^{m+1}\left|\sigma_{*}^{\theta} a_{r, s}(x)\right|^{p} d x .
$$

Here we have to change slightly the definition of $A_{K, N, n}(x)$, set $A_{K, N, n, r, s}(x)=$ $A_{K, N, n}(x+n / \beta)$. More exactly, similarly to (15) we have

$$
\int_{m}^{m+1}\left|\sigma_{*}^{\theta} a_{r, s}(x)\right|^{p} d x
$$




$$
\begin{aligned}
\leq & \sum_{n \in \mathbb{Z}^{2}} \int_{m}^{m+1} \sup _{K \in \mathbb{N}^{2}, N \in \mathbb{N}_{\tau}^{2}} \mid \int_{I} a_{r, s}(t) \sum_{\left|k_{1}\right| \leq K_{1}} \sum_{\left|k_{2}\right| \leq K_{2}} N_{1} \hat{\theta}_{1}\left(N_{1} \beta\left(x_{1}-t_{1}+n_{1} / \beta\right)\right) \\
& \left.N_{2} \hat{\theta}_{2}\left(N_{2} \beta\left(x_{2}-t_{2}+n_{2} / \beta\right)\right) \overline{T_{\alpha k} g(t)} T_{\alpha k} \gamma(x) d t\right|^{p} d x \\
= & \sum_{n \in \mathbb{Z}^{2}} \int_{m}^{m+1} \sup _{K \in \mathbb{N}^{2}, N \in \mathbb{N}_{\tau}^{2}}\left|A_{K, N, n, r, s}(x)\right|^{p} d x .
\end{aligned}
$$

For simplicity we assume that each coordinate of $r-m-n / \beta$ is non-negative, i.e. $r_{l}-m_{l}-n_{l} / \beta \geq 0(l=1,2)$.

Step 1. First assume that $\left|r_{l}-m_{l}-n_{l} / \beta\right| \leq 2(l=1,2)$. Then

$$
\begin{aligned}
\int_{m}^{m+1} & \left|\sigma_{*}^{\theta} a_{r, s}(x)\right|^{p} d x \\
\leq & \sum_{n \in \mathbb{Z}^{2}}\left(\int_{(m, m+1) \cap\left(4 I_{r, s, 1}-n_{1} / \beta\right) \times\left(4 I_{r, s, 2}-n_{2} / \beta\right)}+\int_{(m, m+1) \cap\left(4 I_{r, s, 1}-n_{1} / \beta\right)^{c} \times\left(4 I_{r, s, 2}-n_{2} / \beta\right)}\right. \\
& \left.+\int_{(m, m+1) \cap\left(4 I_{r, s, 1}-n_{1} / \beta\right) \times\left(4 I_{r, s, 2}-n_{2} / \beta\right)^{c}}+\int_{(m, m+1) \cap\left(4 I_{r, s, 1}-n_{1} / \beta\right)^{c} \times\left(4 I_{r, s, 2}-n_{2} / \beta\right)^{c}}\right) \\
& \sup _{K \in \mathbb{N}^{2}, N \in \mathbb{N}_{\tau}^{2}}\left|A_{K, N, n, r, s}(x)\right|^{p} d x .
\end{aligned}
$$

Step 1.1. For the first expression we obtain as in (16) that

$$
\begin{aligned}
\left|A_{K, N, n, r, s}(x)\right| & \leq C_{p} 2^{2 L_{r, s} / p} \sum_{k \in \mathbb{Z}^{2}}\left\|\hat{\theta}_{1}\right\|_{1}\left\|\hat{\theta}_{2}\right\|_{1} \sup _{I_{r, s}}\left|T_{\alpha k} g \| T_{\alpha k} \gamma(x)\right| \\
& \leq C_{p} 2^{2 L_{r, s} / p} \sum_{k \in \mathbb{Z}^{2}}\left\|\hat{\theta}_{1}\right\|_{1}\left\|\hat{\theta}_{2}\right\|_{1} \sup _{(r, r+3 / 2)}\left|T_{\alpha k} g\right| \sup _{(m, m+1)}\left|T_{\alpha k} \gamma\right| .
\end{aligned}
$$

For fixed $r$ and $m$ there are finitely many $n^{\prime} s$ for which $\left|r_{l}-m_{l}-n_{l} / \beta\right| \leq 2$. Hence

$$
\begin{gathered}
\sum_{r \in \mathbb{Z}^{2}} \sum_{s=0}^{\infty}\left|\mu_{r, s}\right|^{p} \sum_{n \in \mathbb{Z}^{2}} \mathbf{1}_{\left\{\left|r_{1}-m_{1}-n_{1} / \beta\right| \leq 2\right\}} \mathbf{1}_{\left\{\left|r_{2}-m_{2}-n_{2} / \beta\right| \leq 2\right\}} \\
\int_{(m, m+1) \cap\left(4 I_{r, s, 1}-n_{1} / \beta\right) \times\left(4 I_{r, s, 2}-n_{2} / \beta\right)} \sup _{K \in \mathbb{N}^{2}, N \in \mathbb{N}_{\tau}^{2}}\left|A_{K, N, n, r, s}(x)\right|^{p} d x \\
\leq C_{p}\left\|\hat{\theta}_{1}\right\|_{1}^{p}\left\|\hat{\theta}_{2}\right\|_{1}^{p} \sum_{r \in \mathbb{Z}^{2}} \sum_{s=0}^{\infty}\left|\mu_{r, s}\right|^{p} \sum_{k \in \mathbb{Z}^{2}} \sup _{(r, r+3 / 2)}\left|T_{\alpha k} g\right|^{p} \sup _{(m, m+1)}\left|T_{\alpha k} \gamma\right|^{p} \\
\leq C_{p}\left\|\hat{\theta}_{1}\right\|_{1}^{p}\left\|\hat{\theta}_{2}\right\|_{1}^{p}\|f\|_{W\left(h_{p}, \ell_{\infty}\right)}^{p} \sum_{r \in \mathbb{Z}^{2}} \sum_{k \in \mathbb{Z}^{2}} \sup _{(r, r+3 / 2)}\left|T_{\alpha k} g\right|^{p} \sup _{(m, m+1)}\left|T_{\alpha k} \gamma\right|^{p} \\
\leq C_{p}\left\|\hat{\theta}_{1}\right\|_{1}^{p}\left\|\hat{\theta}_{2}\right\|_{1}^{p}\|g\|_{W\left(L_{\infty}, \ell_{p}\right)}^{p}\|\gamma\|_{W\left(L_{\infty}, \ell_{p}\right)}^{p}\|f\|_{W\left(h_{p}, \ell_{\infty}\right)}^{p}
\end{gathered}
$$


We split the supremum again into two parts:

$$
\begin{aligned}
& \sup _{K \in \mathbb{N}^{2}, N \in \mathbb{N}_{\tau}^{2}}\left|A_{K, N, n, r, s}(x)\right| \\
& \leq \sup _{K \in \mathbb{N}^{2}, N_{1}, N_{2} \geq 2^{L r, s-\tau}}\left|A_{K, N, n, r, s}(x)\right|+\sup _{\substack{K \in \mathbb{N}^{2}, N \in \mathbb{N}_{\tau}^{2} \\
N_{1}, N_{2}<2^{L r, s}}}\left|A_{K, N, n, r, s}(x)\right| .
\end{aligned}
$$

Step 1.2. Let $x \in(m, m+1) \cap\left(4 I_{r, s, 1}-n_{1} / \beta\right)^{c} \times\left(4 I_{r, s, 2}-n_{2} / \beta\right)$ and $N_{1}, N_{2} \geq 2^{L_{r, s}-\tau}$. If $x_{1} \in B\left(t_{r, s, 1}-n_{1} / \beta,\left(i_{1}+1\right) 2^{-L_{r, s}}\right) \backslash B\left(t_{r, s, 1}-n_{1} / \beta, i_{1} 2^{-L_{r, s}}\right)$ $\left(i_{1} \geq 1\right)$ then

$$
\left|x_{1}-t_{1}+n_{1} / \beta\right| \geq C i_{1} 2^{-L_{r, s}}
$$

where $t_{r, s}$ is the center of $I_{r, s}$. Similarly to (17),

$$
\left|A_{K, N, n, r, s}(x)\right| \leq C_{p} 2^{2 L_{r, s} / p}\left\|\hat{\theta}_{2}\right\|_{1} i_{1}^{-\delta_{1}} \sum_{k \in \mathbb{Z}^{2}} \sup _{(r, r+3 / 2)}\left|T_{\alpha k} g\right| \sup _{(m, m+1)}\left|T_{\alpha k} \gamma\right| .
$$

Then

$$
\begin{aligned}
& \int_{(m, m+1) \cap\left(4 I_{r, s, 1}-n_{1} / \beta\right)^{c} \times\left(4 I_{r, s, 2}-n_{2} / \beta\right)} \sup _{K \in \mathbb{N}^{2}, N_{1}, N_{2} \geq 2^{L, s-\tau}}\left|A_{K, N, n, r, s}(x)\right|^{p} d x \\
& \leq \sum_{i_{1}=1}^{\infty} \int_{B\left(t_{r, s, 1}-n_{1} / \beta,\left(i_{1}+1\right) 2^{-L r, s}\right) \backslash B\left(t_{r, s, 1}-n_{1} / \beta, i_{1} 2^{-L r, s}\right)} \\
& \int_{4 I_{r, s, 2}-n_{2} / \beta} \sup _{K \in \mathbb{N}^{2}, N_{1}, N_{2} \geq 2^{L r, s}-\tau}\left|A_{K, N, n, r, s}(x)\right|^{p} d x \\
& \leq C_{p}\left\|\hat{\theta}_{2}\right\|_{1}^{p} \sum_{k \in \mathbb{Z}^{2}} \sup _{(r, r+3 / 2)}\left|T_{\alpha k} g\right|^{p} \sup _{(m, m+1)}\left|T_{\alpha k} \gamma\right|^{p}
\end{aligned}
$$

and so

$$
\begin{aligned}
& \sum_{r \in \mathbb{Z}^{2}} \sum_{s=0}^{\infty}\left|\mu_{r, s}\right|^{p} \sum_{n \in \mathbb{Z}^{2}} \mathbf{1}_{\left\{\left|r_{1}-m_{1}-n_{1} / \beta\right| \leq 2\right\}} \mathbf{1}_{\left\{\left|r_{2}-m_{2}-n_{2} / \beta\right| \leq 2\right\}} \\
& \quad \int_{(m, m+1) \cap\left(4 I_{r, s, 1}-n_{1} / \beta\right)^{c} \times\left(4 I_{r, s, 2}-n_{2} / \beta\right)} \sup _{K \in \mathbb{N}^{2}, N_{1}, N_{2} \geq 2^{L r, s-\tau}}\left|A_{K, N, n, r, s}(x)\right|^{p} d x \\
& \leq C_{p}\left\|\hat{\theta}_{2}\right\|_{1}^{p}\|g\|_{W\left(L_{\infty}, \ell_{p}\right)}^{p}\|\gamma\|_{W\left(L_{\infty}, \ell_{p}\right)}^{p}\|f\|_{W\left(h_{p}, \ell_{\infty}\right)}^{p} .
\end{aligned}
$$

Remark that (21) and (22) work for both 1 and 2 type atoms, because $I_{r, s} \subset[r, r+3 / 2)$.

Step 1.3. Let $x \in(m, m+1) \cap\left(4 I_{r, s, 1}-n_{1} / \beta\right)^{c} \times\left(4 I_{r, s, 2}-n_{2} / \beta\right)$ and $N_{1}, N_{2}<2^{L_{r, s}}$. If $a$ is a type 2 atom then

$$
\sup _{\substack{K \in \mathbb{N}^{2}, N \in \mathbb{N}_{\tau}^{2} \\ N_{1}, N_{2}<2^{L r, s}}}\left|A_{K, N, n, r, s}(x)\right|=0 .
$$


So we may assume that $a$ is a type 1 atom. We define $B_{K, N, n, r, s}$ and $C_{K, N, n, r, s}$ similarly to (19), $B_{K, N, n, r, s}(x)=B_{K, N, n}(x+n / \beta), C_{K, N, n, r, s}(x)=C_{K, N, n}(x+$ $n / \beta)$. Then

$$
\begin{aligned}
& \left|B_{K, N, n, r, s}(x)\right|,\left|C_{K, N, n, r, s}(x)\right| \\
& \quad \leq C_{p} 2^{2 L_{r, s} / p}\left\|\hat{\theta}_{2}\right\|_{1} i_{1}^{-\delta_{1}} \sum_{k \in \mathbb{Z}^{2}} \sup _{(r, r+3 / 2)}\left|T_{\alpha k} g\right| \sup _{(m, m+1)}\left|T_{\alpha k} \gamma\right|,
\end{aligned}
$$

if $x_{1} \in B\left(t_{r, s, 1}-n_{1} / \beta,\left(i_{1}+1\right) 2^{-L_{r, s}}\right) \backslash B\left(t_{r, s, 1}-n_{1} / \beta, i_{1} 2^{-L_{r, s}}\right)$ and

$$
\begin{gathered}
\sum_{r \in \mathbb{Z}^{2}} \sum_{s=0}^{\infty}\left|\mu_{r, s}\right|^{p} \sum_{n \in \mathbb{Z}^{2}} \mathbf{1}_{\left\{\left|r_{1}-m_{1}-n_{1} / \beta\right| \leq 2\right\}} \mathbf{1}_{\left\{\left|r_{2}-m_{2}-n_{2} / \beta\right| \leq 2\right\}} \\
\int_{(m, m+1) \cap\left(4 I_{r, s, 1}-n_{1} / \beta\right)^{c} \times\left(4 I_{r, s, 2}-n_{2} / \beta\right)} \sup _{\substack{K \in \mathbb{N}^{2}, N \in \mathbb{N}_{T}^{2} \\
N_{1}, N_{2}<L^{L}, s}}\left|A_{K, N, n, r, s}(x)\right|^{p} d x \\
\leq C_{p}\left\|\hat{\theta}_{2}\right\|_{1}^{p}\|g\|_{W\left(L_{\infty}, \ell_{p}\right)}^{p}\|\gamma\|_{W\left(L_{\infty}, \ell_{p}\right)}^{p}\|f\|_{W\left(h_{p}, \ell_{\infty}\right)}^{p} .
\end{gathered}
$$

Step 1.4. The integration over $(m, m+1) \cap\left(4 I_{r, s, 1}-n_{1} / \beta\right) \times\left(4 I_{r, s, 2}-n_{2} / \beta\right)^{c}$ is similar.

Step 1.5. Let $x \in(m, m+1) \cap\left(4 I_{r, s, 1}-n_{1} / \beta\right)^{c} \times\left(4 I_{r, s, 2}-n_{2} / \beta\right)^{c}$ and $N_{1}, N_{2} \geq 2^{L_{r, s}-\tau}$. If $x_{1} \in B\left(t_{r, s, 1}-n_{1} / \beta,\left(i_{1}+1\right) 2^{-L_{r, s}}\right) \backslash B\left(t_{r, s, 1}-n_{1} / \beta, i_{1} 2^{-L_{r, s}}\right)$ and $x_{2} \in B\left(t_{r, s, 2}-n_{2} / \beta,\left(i_{2}+1\right) 2^{-L_{r, s}}\right) \backslash B\left(t_{r, s, 2}-n_{2} / \beta, i_{2} 2^{-L_{r, s}}\right)\left(i_{1}, i_{2} \geq 1\right)$ then

$$
\left|A_{K, N, n, r, s}(x)\right| \leq C_{p} 2^{2 L_{r, s} / p} i_{1}^{-\delta_{1}} i_{2}^{-\delta_{2}} \sum_{k \in \mathbb{Z}^{2}} \sup _{(r, r+3 / 2)}\left|T_{\alpha k} g\right| \sup _{(m, m+1)}\left|T_{\alpha k} \gamma\right|
$$

and

$$
\begin{gathered}
\sum_{r \in \mathbb{Z}^{2}} \sum_{s=0}^{\infty}\left|\mu_{r, s}\right|^{p} \sum_{n \in \mathbb{Z}^{2}} \mathbf{1}_{\left\{\left|r_{1}-m_{1}-n_{1} / \beta\right| \leq 2\right\}} \mathbf{1}_{\left\{\left|r_{2}-m_{2}-n_{2} / \beta\right| \leq 2\right\}} \\
\int_{(m, m+1) \cap\left(4 I_{r, s, 1}-n_{1} / \beta\right)^{c} \times\left(4 I_{r, s, 2}-n_{2} / \beta\right)^{c}} \sup _{K \in \mathbb{N}^{2}, N_{1}, N_{2} \geq 2^{L_{r, s}-\tau}}\left|A_{K, N, n, r, s}(x)\right|^{p} d x \\
\leq C_{p}\|g\|_{W\left(L_{\infty}, \ell_{p}\right)}^{p}\|\gamma\|_{W\left(L_{\infty}, \ell_{p}\right)}^{p}\|f\|_{W\left(h_{p}, \ell_{\infty}\right)}^{p} .
\end{gathered}
$$

Step 1.6. Let $x \in(m, m+1) \cap\left(4 I_{r, s, 1}-n_{1} / \beta\right)^{c} \times\left(4 I_{r, s, 2}-n_{2} / \beta\right)^{c}$ and $N_{1}, N_{2}<2^{L_{r, s}}$. We define $B_{l, K, N, n, r, s}$ and $C_{l, K, N, n, r, s}$ as in the proof of Theorem $3, B_{l, K, N, n, r, s}(x)=B_{l, K, N, n}(x+n / \beta), C_{l, K, N, n, r, s}(x)=C_{l, K, N, n}(x+n / \beta), l=1,2$. Then

$$
\left|B_{1, K, N, n, r, s}(x)\right| \leq C_{p} 2^{2 L_{r, s} / p} i_{1}^{-\delta_{1}} i_{2}^{-\delta_{2}} \sum_{k \in \mathbb{Z}^{2}} \sup _{(r, r+3 / 2)}\left|T_{\alpha k} g\right| \sup _{(m, m+1)}\left|T_{\alpha k} \gamma\right|,
$$




$$
\begin{aligned}
& \left|B_{2, K, N, n, r, s}(x)\right|,\left|C_{1, K, N, n, r, s}(x)\right| \\
& \leq C_{p} 2^{2 L_{r, s} / p} i_{1}^{-\delta_{1} \eta_{1}} i_{2}^{-\delta_{2} \eta_{2}} \sum_{k \in \mathbb{Z}^{2}} \sup _{(r, r+3 / 2)}\left|T_{\alpha k} g\right| \sup _{(m, m+1)}\left|T_{\alpha k} \gamma\right|, \\
& \left|C_{2, K, N, n, r, s}(x)\right| \leq C_{p} 2^{2 L_{r, s} / p} i_{1}^{-\delta_{1}} i_{2}^{-\delta_{2}} \sum_{k \in \mathbb{Z}^{2}} \sup _{(r, r+3 / 2)}\left|\sum_{i_{1}+i_{2}=2} \partial_{1}^{i_{1}} \partial_{2}^{i_{2}} T_{\alpha k} g\right| \sup _{(m, m+1)}\left|T_{\alpha k} \gamma\right|,
\end{aligned}
$$

if $x_{1} \in B\left(t_{r, s, 1}-n_{1} / \beta,\left(i_{1}+1\right) 2^{-L_{r, s}}\right) \backslash B\left(t_{r, s, 1}-n_{1} / \beta, i_{1} 2^{-L_{r, s}}\right)$ and $x_{2} \in B\left(t_{r, s, 2}-\right.$ $\left.n_{2} / \beta,\left(i_{2}+1\right) 2^{-L_{r, s}}\right) \backslash B\left(t_{r, s, 2}-n_{2} / \beta, i_{2} 2^{-L_{r, s}}\right)\left(i_{1}, i_{2} \geq 1\right)$. Hence

$$
\begin{aligned}
& \sum_{r \in \mathbb{Z}^{2}} \sum_{s=0}^{\infty}\left|\mu_{r, s}\right|^{p} \sum_{n \in \mathbb{Z}^{2}} \mathbf{1}_{\left\{\left|r_{1}-m_{1}-n_{1} / \beta\right| \leq 2\right\}} \mathbf{1}_{\left\{\left|r_{2}-m_{2}-n_{2} / \beta\right| \leq 2\right\}} \\
& \int_{(m, m+1) \cap\left(4 I_{r, s, 1}-n_{1} / \beta\right)^{c} \times\left(4 I_{r, s, 2}-n_{2} / \beta\right)^{c}} \sup _{\substack{K \in \mathbb{N}^{2}, N \in \mathbb{N}_{T}^{2} \\
N_{1}, N_{2}<L^{L r, s}}}\left|A_{K, N, n, r, s}(x)\right|^{p} d x \\
& \leq C_{p}\left\|\sum_{i_{1}+i_{2}=0,2} \partial_{1}^{i_{1}} \partial_{2}^{i_{2}} T_{\alpha k} g\right\|_{W\left(L_{\infty}, \ell_{p}\right)}^{p}\|\gamma\|_{W\left(L_{\infty}, \ell_{p}\right)}^{p}\|f\|_{W\left(h_{p}, \ell_{\infty}\right)}^{p} .
\end{aligned}
$$

Step 2. Secondly assume that $\left|r_{1}-m_{1}-n_{1} / \beta\right|>2$ and $\left|r_{2}-m_{2}-n_{2} / \beta\right| \leq 2$. Observe that if $x_{1} \in\left(m_{1}, m_{1}+1\right)$ and $t \in I_{r, s}$ then

$\left|t_{1}-x_{1}-n_{1} / \beta\right| \geq\left|r_{1}-m_{1}-1-n_{1} / \beta\right| \geq\left|r_{1}-m_{1}-n_{1} / \beta\right|-1 \geq \frac{\left|r_{1}-m_{1}-n_{1} / \beta\right|}{2}$.

In this case

$$
\begin{aligned}
& \int_{m}^{m+1}\left|\sigma_{*}^{\theta} a_{r, s}(x)\right|^{p} d x \\
& \quad \leq \sum_{n \in \mathbb{Z}^{2}}\left(\int_{(m, m+1) \cap \mathbb{R} \times\left(4 I_{r, s, 2}-n_{2} / \beta\right)}+\int_{(m, m+1) \cap \mathbb{R} \times\left(4 I_{r, s, 2}-n_{2} / \beta\right)^{c}} \sup _{K \in \mathbb{N}^{2}, N \in \mathbb{N}_{\tau}^{2}}\left|A_{K, N, n, r, s}(x)\right|^{p} d x\right) .
\end{aligned}
$$

Step 2.1. Let $x \in(m, m+1) \cap \mathbb{R} \times\left(4 I_{r, s, 2}-n_{2} / \beta\right)$ and $N_{1}, N_{2} \geq 2^{L_{r, s}-\tau}$. We obtain

$$
\begin{aligned}
\left|A_{K, N, n, r, s}(x)\right|= & \mid \int_{I} a_{r, s}(t) \sum_{\left|k_{1}\right| \leq K_{1}} \sum_{\left|k_{2}\right| \leq K_{2}} N_{1} \hat{\theta}_{1}\left(N_{1} \beta\left(x_{1}-t_{1}+n_{1} / \beta\right)\right) \\
& N_{2} \hat{\theta}_{2}\left(N_{2} \beta\left(x_{2}-t_{2}+n_{2} / \beta\right)\right) \overline{T_{\alpha k} g(t)} T_{\alpha k} \gamma(x) d t \mid \\
\leq & C_{p} 2^{2 L_{r, s} / p-L_{r, s}} N_{1}^{1-\delta_{1}} \sum_{k \in \mathbb{Z}^{2}}\left|r_{1}-m_{1}-n_{1} / \beta\right|^{-\delta_{1}}\left\|\hat{\theta}_{2}\right\|_{1} \\
& \sup _{(r, r+3 / 2)}\left|T_{\alpha k} g\right| \sup _{(m, m+1)}\left|T_{\alpha k} \gamma\right|
\end{aligned}
$$




$$
\begin{aligned}
\leq & C_{p} 2^{2 L_{r, s} / p-\delta_{1} L_{r, s}} \sum_{k \in \mathbb{Z}^{2}}\left|r_{1}-m_{1}-n_{1} / \beta\right|^{-\delta_{1}}\left\|\hat{\theta}_{2}\right\|_{1} \\
\sup _{(r, r+3 / 2)}\left|T_{\alpha k} g\right| & \sup _{(m, m+1)}\left|T_{\alpha k} \gamma\right|
\end{aligned}
$$

and

$$
\begin{aligned}
& \sum_{r \in \mathbb{Z}^{2}} \sum_{s=0}^{\infty}\left|\mu_{r, s}\right|^{p} \sum_{n \in \mathbb{Z}^{2}} \mathbf{1}_{\left\{\left|r_{1}-m_{1}-n_{1} / \beta\right|>2\right\}} \mathbf{1}_{\left\{\left|r_{2}-m_{2}-n_{2} / \beta\right| \leq 2\right\}} \sup _{(m, m+1) \cap \mathbb{R} \times\left(4 I_{r, s, 2}-n_{2} / \beta\right)}\left|A_{K, N, n, r, s}(x)\right|^{p} d x \\
& \int_{K \in \mathbb{N}^{2}, N_{1}, N_{2} \geq 2^{L r, s}-\tau} \\
& \leq C_{p}\left\|\hat{\theta}_{2}\right\|_{1}^{p} \sum_{r \in \mathbb{Z}^{2}} \sum_{s=0}^{\infty}\left|\mu_{r, s}\right|^{p} \sum_{n_{1} \in \mathbb{Z}} \mathbf{1}_{\left\{\left|r_{1}-m_{1}-n_{1} / \beta\right|>2\right\}} 2^{L_{r, s}-\delta_{1} L_{r, s} p}\left|r_{1}-m_{1}-n_{1} / \beta\right|^{-\delta_{1} p} \\
& \sum_{k \in \mathbb{Z}^{2}} \sup _{(r, r+3 / 2)}\left|T_{\alpha k} g\right|^{p} \sup _{(m, m+1)}\left|T_{\alpha k} \gamma\right|^{p} \\
& \leq C_{p}\left\|\hat{\theta}_{2}\right\|_{1}^{p}\|f\|_{W\left(h_{p}, \ell_{\infty}\right)}^{p} \sum_{r \in \mathbb{Z}^{2}} \sum_{k \in \mathbb{Z}^{2}(r, r+3 / 2)} \sup _{\left|T_{\alpha k} g\right|^{p} \sup _{(m, m+1)}\left|T_{\alpha k} \gamma\right|^{p}}^{\leq} \\
& C_{p}\left\|\hat{\theta}_{2}\right\|_{1}^{p}\|g\|_{W\left(L_{\infty}, \ell_{p}\right)}^{p}\|\gamma\|_{W\left(L_{\infty}, \ell_{p}\right)}^{p}\|f\|_{W\left(h_{p}, \ell_{\infty}\right)}^{p} .
\end{aligned}
$$

Step 2.2. Let $x \in(m, m+1) \cap \mathbb{R} \times\left(4 I_{r, s, 2}-n_{2} / \beta\right)$ and $N_{1}, N_{2}<2^{L_{r, s}}$. In this case

$$
\begin{aligned}
\left|A_{K, N, n, r, s}(x)\right| \leq & C_{p} 2^{2 L_{r, s} / p-2 L_{r, s}} N_{1}^{1-\delta_{1}} N_{2} \sum_{k \in \mathbb{Z}^{2}}\left|r_{1}-m_{1}-n_{1} / \beta\right|^{-\delta_{1}}\left\|\hat{\theta}_{2}\right\|_{\infty} \\
& \sup _{(r, r+3 / 2)}\left|T_{\alpha k} g\right| \sup _{(m, m+1)}\left|T_{\alpha k} \gamma\right| \\
\leq & C_{p} 2^{2 L_{r, s} / p-\delta_{1} L_{r, s}} \sum_{k \in \mathbb{Z}^{2}}\left|r_{1}-m_{1}-n_{1} / \beta\right|^{-\delta_{1}} \\
& \sup _{(r, r+3 / 2)}\left|T_{\alpha k} g\right| \sup _{(m, m+1)}\left|T_{\alpha k} \gamma\right|
\end{aligned}
$$

and

$$
\begin{aligned}
\sum_{r \in \mathbb{Z}^{2}} \sum_{s=0}^{\infty}\left|\mu_{r, s}\right|^{p} \sum_{n \in \mathbb{Z}^{2}} \mathbf{1}_{\left\{\left|r_{1}-m_{1}-n_{1} / \beta\right|>2\right\}} \mathbf{1}_{\left\{\left|r_{2}-m_{2}-n_{2} / \beta\right| \leq 2\right\}} & \sup _{\substack{K \in \mathbb{N}^{2}, N \in \mathbb{N}_{T}^{2} \\
N_{1}, N_{2}<2^{L}, s}}\left|A_{K, N, n, r, s}(x)\right|^{p} d x \\
& \int_{(m, m+1) \cap \mathbb{R} \times\left(4 I_{r, s, 2}-n_{2} / n_{2}\right.} \\
\leq & C_{p}\|g\|_{W\left(L_{\infty}, \ell_{p}\right)}^{p}\|\gamma\|_{W\left(L_{\infty}, \ell_{p}\right)}^{p}\|f\|_{W\left(h_{p}, \ell_{\infty}\right)}^{p}
\end{aligned}
$$

as before. 
Step 2.3. Let $x \in(m, m+1) \cap \mathbb{R} \times\left(4 I_{r, s, 2}-n_{2} / \beta\right)^{c}$ and $N_{1}, N_{2} \geq 2^{L_{r, s}-\tau}$. If $x_{2} \in B\left(t_{r, s, 2}-n_{2} / \beta,\left(i_{2}+1\right) 2^{-L_{r, s}}\right) \backslash B\left(t_{r, s, 2}-n_{2} / \beta, i_{2} 2^{-L_{r, s}}\right)\left(i_{2} \geq 1\right)$ then

$$
\begin{aligned}
\leq & C_{p} 2^{2 L_{r, s} / p-2 L_{r, s}+L_{r, s} \delta_{2}} N_{1}^{1-\delta_{1}} N_{2}^{1-\delta_{2}} i_{2}^{-\delta_{2}} \sum_{k \in \mathbb{Z}^{2}}\left|r_{1}-m_{1}-n_{1} / \beta\right|^{-\delta_{1}} \\
& \sup _{(r, r+3 / 2)}\left|T_{\alpha k} g\right| \sup _{(m, m+1)}\left|T_{\alpha k} \gamma\right| \\
\leq & C_{p} 2^{2 L_{r, s} / p-\delta_{1} L_{r, s}} i_{2}^{-\delta_{2}} \sum_{k \in \mathbb{Z}^{2}}\left|r_{1}-m_{1}-n_{1} / \beta\right|^{-\delta_{1}} \\
& \sup _{(r, r+3 / 2)}\left|T_{\alpha k} g\right| \sup _{(m, m+1)}\left|T_{\alpha k} \gamma\right|
\end{aligned}
$$

which implies

$$
\begin{aligned}
\sum_{r \in \mathbb{Z}^{2}} & \sum_{s=0}^{\infty}\left|\mu_{r, s}\right|^{p} \sum_{n \in \mathbb{Z}^{2}} \mathbf{1}_{\left\{\left|r_{1}-m_{1}-n_{1} / \beta\right|>2\right\}} \mathbf{1}_{\left\{\left|r_{2}-m_{2}-n_{2} / \beta\right| \leq 2\right\}} \\
& \int_{(m, m+1) \cap \mathbb{R} \times\left(4 I_{r, s, 2}-n_{2} / \beta\right)^{c}} \sup _{K \in \mathbb{N}^{2}, N_{1}, N_{2} \geq 2^{L r, s-\tau}}\left|A_{K, N, n, r, s}(x)\right|^{p} d x \\
\leq & C_{p}\|g\|_{W\left(L_{\infty}, \ell_{p}\right)}^{p}\|\gamma\|_{W\left(L_{\infty}, \ell_{p}\right)}^{p}\|f\|_{W\left(h_{p}, \ell_{\infty}\right)}^{p} .
\end{aligned}
$$

Step 2.4. Let $x \in(m, m+1) \cap \mathbb{R} \times\left(4 I_{r, s, 2}-n_{2} / \beta\right)^{c}$ and $N_{1}, N_{2}<2^{L_{r, s}-\tau}$. If $x_{2} \in B\left(t_{r, s, 2}-n_{2} / \beta,\left(i_{2}+1\right) 2^{-L_{r, s}}\right) \backslash B\left(t_{r, s, 2}-n_{2} / \beta, i_{2} 2^{-L_{r, s}}\right)\left(i_{2} \geq 1\right)$ then

$$
\begin{aligned}
\leq & C_{p} 2^{2 L_{r, s} / p-4 L_{r, s}+L_{r, s} \delta_{2}} N_{1}^{2-\delta_{1}} N_{2}^{2-\delta_{2}} i_{2}^{-\delta_{2}} \sum_{k \in \mathbb{Z}^{2}}\left|r_{1}-m_{1}-n_{1} / \beta\right|^{-\delta_{1}} \\
& \sup _{(r, r+3 / 2)}\left|T_{\alpha k} g\right| \sup _{(m, m+1)}\left|T_{\alpha k} \gamma\right| \\
\leq & C_{p} 2^{2 L_{r, s} / p-\delta_{1} L_{r, s}} i_{2}^{-\delta_{2}} \sum_{k \in \mathbb{Z}^{2}}\left|r_{1}-m_{1}-n_{1} / \beta\right|^{-\delta_{1}} \\
& \sup _{(r, r+3 / 2)}\left|T_{\alpha k} g\right| \sup _{(m, m+1)}\left|T_{\alpha k} \gamma\right|
\end{aligned}
$$

and

$$
\begin{aligned}
& \left|B_{2, K, N, n, r, s}(x)\right| \\
\leq & C_{p} 2^{2 L_{r, s} / p-3 L_{r, s}+L_{r, s} \delta_{2} \eta_{2}} N_{1}^{2-\delta_{1} \eta_{1}} N_{2}^{1-\delta_{2} \eta_{2}} i_{2}^{-\delta_{2} \eta_{2}} \sum_{k \in \mathbb{Z}^{2}}\left|r_{1}-m_{1}-n_{1} / \beta\right|^{-\delta_{1} \eta_{1}} \\
& \sup _{(r, r+3 / 2)}\left|T_{\alpha k} g\right| \sup _{(m, m+1)}\left|T_{\alpha k} \gamma\right| \\
\leq & C_{p} 2^{2 L_{r, s} / p-\delta_{1} \eta_{1} L_{r, s}} i_{2}^{-\delta_{2} \eta_{2}} \sum_{k \in \mathbb{Z}^{2}}\left|r_{1}-m_{1}-n_{1} / \beta\right|^{-\delta_{1} \eta_{1}} \\
& \sup _{(r, r+3 / 2)}\left|T_{\alpha k} g\right| \sup _{(m, m+1)}\left|T_{\alpha k} \gamma\right| .
\end{aligned}
$$


The term $C_{1, K, N, n, r, s}$ can be handled as $B_{2, K, N, n, r, s}$. Moreover,

$$
\begin{aligned}
\left|C_{2, K, N, n, r, s}(x)\right| \leq & C_{p} 2^{2 L_{r, s} / p-4 L_{r, s}+L_{r, s} \delta_{2}} N_{1}^{1-\delta_{1}} N_{2}^{1-\delta_{2}} i_{2}^{-\delta_{2}} \sum_{k \in \mathbb{Z}^{2}}\left|r_{1}-m_{1}-n_{1} / \beta\right|^{-\delta_{1}} \\
& \sup _{(r, r+3 / 2)}\left|\sum_{i_{1}+i_{2}=2} \partial_{1}^{i_{1}} \partial_{2}^{i_{2}} T_{\alpha k} g\right| \sup _{(m, m+1)}\left|T_{\alpha k} \gamma\right| \\
\leq & C_{p} 2^{2 L_{r, s} / p-2 L_{r, s}} i_{2}^{-\delta_{2}} \sum_{k \in \mathbb{Z}^{2}}\left|r_{1}-m_{1}-n_{1} / \beta\right|^{-\delta_{1}} \\
& \sup _{(r, r+3 / 2)}\left|\sum_{i_{1}+i_{2}=2} \partial_{1}^{i_{1}} \partial_{2}^{i_{2}} T_{\alpha k} g\right| \sup _{(m, m+1)}\left|T_{\alpha k} \gamma\right| .
\end{aligned}
$$

Note that $p>1 / 2$. From this it follows that

$$
\begin{aligned}
\sum_{r \in \mathbb{Z}^{2}} & \sum_{s=0}^{\infty}\left|\mu_{r, s}\right|^{p} \sum_{n \in \mathbb{Z}^{2}} \mathbf{1}_{\left\{\left|r_{1}-m_{1}-n_{1} / \beta\right|>2\right\}} \mathbf{1}_{\left\{\left|r_{2}-m_{2}-n_{2} / \beta\right| \leq 2\right\}} \\
& \int_{(m, m+1) \cap \mathbb{R} \times\left(4 I_{r, s, 2}-n_{2} / \beta\right)^{c}} \sup _{\substack{K \in \mathbb{N}^{2}, N \in \mathbb{N}_{T}^{2} \\
N_{1}, N_{2}<2^{L}, s}}\left|A_{K, N, n, r, s}(x)\right|^{p} d x \\
\leq & C_{p}\left\|\sum_{i_{1}+i_{2}=0,2} \partial_{1}^{i_{1}} \partial_{2}^{i_{2}} T_{\alpha k} g\right\|_{W\left(L_{\infty}, \ell_{p}\right)}^{p}\|\gamma\|_{W\left(L_{\infty}, \ell_{p}\right)}^{p}\|f\|_{W\left(h_{p}, \ell_{\infty}\right)}^{p} .
\end{aligned}
$$

Step 3. The case $\left|r_{1}-m_{1}-n_{1} / \beta\right| \leq 2$ and $\left|r_{2}-m_{2}-n_{2} / \beta\right|>2$ can be handled similarly to Step 2.

Step 4. Finally assume that $\left|r_{1}-m_{1}-n_{1} / \beta\right|>2$ and $\left|r_{2}-m_{2}-n_{2} / \beta\right|>2$. Now

$$
\int_{m}^{m+1}\left|\sigma_{*}^{\theta} a_{r, s}(x)\right|^{p} d x \leq \sum_{n \in \mathbb{Z}^{2}} \int_{(m, m+1)} \sup _{K \in \mathbb{N}^{2}, N \in \mathbb{N}_{\tau}^{2}}\left|A_{K, N, n, r, s}(x)\right|^{p} d x .
$$

Step 4.1. Let $x \in(m, m+1)$ and $N_{1}, N_{2} \geq 2^{L_{r, s}-\tau}$. We obtain

$$
\begin{aligned}
\left|A_{K, N, n, r, s}(x)\right| \leq & C_{p} 2^{2 L_{r, s} / p-2 L_{r, s}} N_{1}^{1-\delta_{1}} N_{2}^{1-\delta_{2}} \sum_{k \in \mathbb{Z}^{2}}\left|r_{1}-m_{1}-n_{1} / \beta\right|^{-\delta_{1}} \\
& \left|r_{2}-m_{2}-n_{2} / \beta\right|^{-\delta_{2}} \sup _{(r, r+3 / 2)}\left|T_{\alpha k} g\right| \sup _{(m, m+1)}\left|T_{\alpha k} \gamma\right| \\
\leq & C_{p} 2^{2 L_{r, s} / p-\delta_{1} L_{r, s}-\delta_{2} L_{r, s}} \sum_{k \in \mathbb{Z}^{2}}\left|r_{1}-m_{1}-n_{1} / \beta\right|^{-\delta_{1}} \\
& \left|r_{2}-m_{2}-n_{2} / \beta\right|^{-\delta_{2}} \sup _{(r, r+3 / 2)}\left|T_{\alpha k} g\right| \sup _{(m, m+1)}\left|T_{\alpha k} \gamma\right|
\end{aligned}
$$

and

$$
\sum_{r \in \mathbb{Z}^{2}} \sum_{s=0}^{\infty}\left|\mu_{r, s}\right|^{p} \sum_{n \in \mathbb{Z}^{2}} \mathbf{1}_{\left\{\left|r_{1}-m_{1}-n_{1} / \beta\right|>2\right\}} \mathbf{1}_{\left\{\left|r_{2}-m_{2}-n_{2} / \beta\right|>2\right\}}
$$




$$
\begin{aligned}
& \int_{(m, m+1)} \sup _{K \in \mathbb{N}^{2}, N_{1}, N_{2} \geq 2^{L_{r, s}-\tau}}\left|A_{K, N, n, r, s}(x)\right|^{p} d x \\
\leq & C_{p}\|g\|_{W\left(L_{\infty}, \ell_{p}\right)}^{p}\|\gamma\|_{W\left(L_{\infty}, \ell_{p}\right)}^{p}\|f\|_{W\left(h_{p}, \ell_{\infty}\right)}^{p} .
\end{aligned}
$$

Step 4.2. If $x \in(m, m+1)$ and $N_{1}, N_{2}<2^{L_{r, s}-\tau}$ then

$$
\begin{aligned}
\left|B_{1, K, N, n, r, s}(x)\right| \leq & C_{p} 2^{2 L_{r, s} / p-4 L_{r, s}} N_{1}^{2-\delta_{1}} N_{2}^{2-\delta_{2}} \sum_{k \in \mathbb{Z}^{2}}\left|r_{1}-m_{1}-n_{1} / \beta\right|^{-\delta_{1}} \\
& \left|r_{2}-m_{2}-n_{2} / \beta\right|^{-\delta_{2}} \sup _{(r, r+3 / 2)}\left|T_{\alpha k} g\right| \sup _{(m, m+1)}\left|T_{\alpha k} \gamma\right| \\
\leq & C_{p} 2^{2 L_{r, s} / p-\delta_{1} L_{r, s}-\delta_{2} L_{r, s}} \sum_{k \in \mathbb{Z}^{2}}\left|r_{1}-m_{1}-n_{1} / \beta\right|^{-\delta_{1}} \\
& \left|r_{2}-m_{2}-n_{2} / \beta\right|^{-\delta_{2}} \sup _{(r, r+3 / 2)}\left|T_{\alpha k} g\right| \sup _{(m, m+1)}\left|T_{\alpha k} \gamma\right| .
\end{aligned}
$$

and

$$
\begin{aligned}
\left|B_{2, K, N, n, r, s}(x)\right| \leq & C_{p} 2^{2 L_{r, s} / p-3 L_{r, s}} N_{1}^{2-\delta_{1} \eta_{1}} N_{2}^{1-\delta_{2} \eta_{2}} \sum_{k \in \mathbb{Z}^{2}}\left|r_{1}-m_{1}-n_{1} / \beta\right|^{-\delta_{1} \eta_{1}} \\
& \left|r_{2}-m_{2}-n_{2} / \beta\right|^{-\delta_{2} \eta_{2}} \sup _{(r, r+3 / 2)}\left|T_{\alpha k} g\right| \sup _{(m, m+1)}\left|T_{\alpha k} \gamma\right| \\
\leq & C_{p} 2^{2 L_{r, s} / p-\delta_{1} \eta_{1} L_{r, s}-\delta_{2} \eta_{2} L_{r, s}} \sum_{k \in \mathbb{Z}^{2}}\left|r_{1}-m_{1}-n_{1} / \beta\right|^{-\delta_{1} \eta_{1}} \\
& \left|r_{2}-m_{2}-n_{2} / \beta\right|^{-\delta_{2} \eta_{2}} \sup _{(r, r+3 / 2)}\left|T_{\alpha k} g\right| \sup _{(m, m+1)}\left|T_{\alpha k} \gamma\right| .
\end{aligned}
$$

The term $C_{1, K, N, n, r, s}$ is similar and

$$
\begin{aligned}
\left|C_{2, K, N, n, r, s}(x)\right| \leq & C_{p} 2^{2 L_{r, s} / p-4 L_{r, s}} N_{1}^{1-\delta_{1}} N_{2}^{1-\delta_{2}} \sum_{k \in \mathbb{Z}^{2}}\left|r_{1}-m_{1}-n_{1} / \beta\right|^{-\delta_{1}} \\
& \left|r_{2}-m_{2}-n_{2} / \beta\right|^{-\delta_{2}} \sup _{(r, r+3 / 2)}\left|\sum_{i_{1}+i_{2}=2} \partial_{1}^{i_{1}} \partial_{2}^{i_{2}} T_{\alpha k} g\right| \sup _{(m, m+1)}\left|T_{\alpha k} \gamma\right| \\
\leq & C_{p} 2^{2 L_{r, s} / p-4 L_{r, s}} \sum_{k \in \mathbb{Z}^{2}}\left|r_{1}-m_{1}-n_{1} / \beta\right|^{-\delta_{1}}\left|r_{2}-m_{2}-n_{2} / \beta\right|^{-\delta_{2}} \\
& \sup _{(r, r+3 / 2)}\left|\sum_{i_{1}+i_{2}=2} \partial_{1}^{i_{1}} \partial_{2}^{i_{2}} T_{\alpha k} g\right| \sup _{(m, m+1)}\left|T_{\alpha k} \gamma\right|
\end{aligned}
$$

which implies

$$
\begin{gathered}
\sum_{r \in \mathbb{Z}^{2}} \sum_{s=0}^{\infty}\left|\mu_{r, s}\right|^{p} \sum_{n \in \mathbb{Z}^{2}} \mathbf{1}_{\left\{\left|r_{1}-m_{1}-n_{1} / \beta\right|>2\right\}} \mathbf{1}_{\left\{\left|r_{2}-m_{2}-n_{2} / \beta\right|>2\right\}} \sup _{\substack{K \in \mathbb{N}^{2}, N \in \mathbb{N}_{2}^{2} \\
N_{1}, N_{2}<2^{L, s}}}\left|A_{K, N, n, r, s}(x)\right|^{p} d x \\
\int_{(m, m+1)}
\end{gathered}
$$




$$
\leq C_{p}\left\|\sum_{i_{1}+i_{2}=0,2} \partial_{1}^{i_{1}} \partial_{2}^{i_{2}} T_{\alpha k} g\right\|_{W\left(L_{\infty}, \ell_{p}\right)}^{p}\|\gamma\|_{W\left(L_{\infty}, \ell_{p}\right)}^{p}\|f\|_{W\left(h_{p}, \ell_{\infty}\right)}^{p} .
$$

This finishes the proof of (12). By complex and real interpolation we can prove (13) and (14) (for the details see Weisz [30]).

\section{References}

[1] J. Benedetto, Gabor representation and wavelets. In D. Colella, editor, Commutative Harmonic Analysis, volume 91 of Contemp. Math., pages 927. American Mathematical Society, Providence, 1989.

[2] J. Bergh and J. Löfström, Interpolation Spaces, an Introduction, Springer, Berlin, 1976.

[3] P. L. Butzer and R. J. Nessel. Fourier Analysis and Approximation. Birkhäuser Verlag, Basel, 1971.

[4] L. Carleson, On convergence and growth of partial sums of Fourier series, Acta Math., 116, 135-157, 1966.

[5] I. Daubechies, Ten Lectures on Wavelets, SIAM, Philadelphia, 1992.

[6] H. G. Feichtinger and F. Weisz, Inversion formulas for the short-time Fourier transform, J. Geom. Anal., 16, 507-521, 2006.

[7] H. G. Feichtinger and F. Weisz, The Segal algebra $\mathbf{S}_{0}\left(\mathbb{R}^{d}\right)$ and norm summability of Fourier series and Fourier transforms, Monatshefte Math., 148, 333-349, 2006.

[8] H. G. Feichtinger and F. Weisz, Wiener amalgams and pointwise summability of Fourier transforms and Fourier series, Math. Proc. Camb. Phil. Soc., 140, 509-536, 2006.

[9] H. G. Feichtinger and F. Weisz, Gabor analysis on Wiener amalgams, Sampl. Theory Signal Image Process, 6, 129-150, 2007.

[10] J. E. Gilbert and J. D. Lakey, On the characterization of the local Hardy space by Gabor frames. In C. Heil, P. E. T. Jorgensen, and D. R. Larson, editors, Wavelets, Frames and Operator Theory, volume 345 of Contemp. Math., pages 153-161. Amer. Math. Soc., Providence, RI, 2004.

[11] D. Goldberg, A local version of real Hardy spaces, Duke Math. J., 46, 27-42, 1979.

[12] L. Grafakos and C. Lennard, Characterization of $L^{p}\left(\mathbf{R}^{n}\right)$ using Gabor frames, J. Fourier Anal. Appl., 7, 101-126, 2001. 
[13] K. Gröchenig, Foundations of Time-Frequency Analysis, Birkhäuser, Boston, 2001.

[14] K. Gröchenig and C. Heil, Gabor meets Littlewood-Paley: Gabor expansions in $L^{p}\left(\mathbf{R}^{d}\right)$, Studia Math., 146, 15-33, 2001.

[15] K. Gröchenig, C. Heil, and K. Okoudjou, Gabor analysis in weighted amalgam spaces, Sampl. Theory Signal Image Process, 1, 225-259, 2002.

[16] K. Gröchenig and M. Leinert, Wiener's lemma for twisted convolution and Gabor frames, J. Amer. Math. Soc., 17, 1-18, 2004.

[17] C. Heil, An introduction to weighted Wiener amalgams. In M. Krishna, R. Radha, and S. Thangavelu, editors, Wavelets and their Applications, pages 183-216. Allied Publishers Private Limited, 2003.

[18] C. E. Heil and D. F. Walnut. Continuous and discrete wavelet transforms. SIAM Rev., 31:628-666, 1989.

[19] I. A. Krishtal and K. A. Okoudjou, Invertibility of the Gabor frame operator on the Wiener amalgam space, J. Approx. Theory, 153, 212-224, 2008.

[20] S. Lu, Four Lectures on Real $H^{p}$ Spaces, World Scientific, Singapore, 1995.

[21] J. Marcinkiewicz and A. Zygmund, On the summability of double Fourier series, Fund. Math., 32, 122-132, 1939.

[22] E. M. Stein, Harmonic Analysis: Real-variable Methods, Orthogonality and Oscillatory Integrals, Princeton Univ. Press, Princeton, N.J., 1993.

[23] E. M. Stein and G. Weiss, Introduction to Fourier Analysis on Euclidean Spaces, Princeton Univ. Press, Princeton, N.J., 1971.

[24] H. Triebel, Theory of Function Spaces I, Akademische Verlagsgesellschaft Geest and Portig K.-G., Leipzig, 1983.

[25] R. M. Trigub and E. S. Belinsky, Fourier Analysis and Approximation of Functions, Kluwer Academic Publishers, Dordrecht, Boston, London, 2004.

[26] D. Walnut, Continuity properties of the Gabor frame operator, J. Math. Anal. Appl., 165, 479-504, 1992.

[27] F. Weisz, Summability of Multi-dimensional Fourier Series and Hardy Spaces, Mathematics and Its Applications. Kluwer Academic Publishers, Dordrecht, Boston, London, 2002.

[28] F. Weisz, Gabor analysis and Hardy spaces, East J. Appr., 15, 1-24, 2009. 
[29] F. Weisz, Pointwise summability of Gabor expansions, J. Fourier Anal. Appl., 15, 463-487, 2009.

[30] F. Weisz, Summability of Gabor expansions and Hardy spaces, Appl. Comput. Harmon. Anal., 30, 288-306, 2011.

[31] A. Zygmund, Trigonometric Series, Cambridge Press, London, 3rd edition, 2002. 